TRANSACTIONS OF THE

AMERICAN MATHEMATICAL SOCIETY

Volume 349, Number 12, December 1997, Pages 4973-5000

S 0002-9947(97)01960-0

\title{
GORENSTEIN ALGEBRAS, SYMMETRIC MATRICES, SELF-LINKED IDEALS, AND SYMBOLIC POWERS
}

\author{
STEVEN KLEIMAN AND BERND ULRICH
}

To David Eisenbud on his fiftieth birthday

\begin{abstract}
Inspired by recent work in the theory of central projections onto hypersurfaces, we characterize self-linked perfect ideals of grade 2 as those with a Hilbert-Burch matrix that has a maximal symmetric subblock. We also prove that every Gorenstein perfect algebra of grade 1 can be presented, as a module, by a symmetric matrix. Both results are derived from the same elementary lemma about symmetrizing a matrix that has, modulo a nonzerodivisor, a symmetric syzygy matrix. In addition, we establish a correspondence, roughly speaking, between Gorenstein perfect algebras of grade 1 that are birational onto their image, on the one hand, and self-linked perfect ideals of grade 2 that have one of the self-linking elements contained in the second symbolic power, on the other hand. Finally, we provide another characterization of these ideals in terms of their symbolic Rees algebras, and we prove a criterion for these algebras to be normal.
\end{abstract}

\section{INTRODUCTION}

A traditional way of studying an algebraic variety is to project it from a general center onto a hypersurface, and conversely a variety with given properties is often constructed by modifying a suitable hypersurface. It is known that the variety is Cohen-Macaulay if and only if, locally, its algebra can be presented, as a module on the target projective space, by a square matrix. If so, then the determinant cuts out the hypersurface, the submaximal minors generate the 'adjoint' ideal (the ideal that induces the conductor), and the adjoint ideal is Cohen-Macaulay of codimension 2; moreover, if the adjoint ideal is radical, then its second symbolic power contains the determinant. Furthermore, the variety is Gorenstein if and only if, via row operations, the matrix can be made symmetric. In this paper, we'll prove in essence that the variety is Gorenstein if and only if the adjoint ideal is self-linked with the determinant as one of the self-linking elements; see Section 3. In Section 2, we'll characterize a self-linked Cohen-Macaulay ideal of codimension 2 as one with a Hilbert-Burch matrix having a maximal symmetric subblock. This result was known before, but not in characteristic 2; moreover, our proof yields, at the same time, the symmetrization result for Gorenstein varieties. In Section 3, we'll

Received by the editors June 2, 1996.

1991 Mathematics Subject Classification. Primary 13C40, 13H10, 13A30, 14E05.

The first author was supported in part by NSF grant 9400918-DMS. It is a pleasure for this author to thank the Mathematical Institute of the University of Copenhagen for its hospitality during the summer of 1995 when this work was completed.

The second author was supported in part by NSF grant DMS-9305832.

(C) 1997 American Mathematical Society 
also characterize, in terms of the symbolic Rees algebra, each such ideal that, in addition, has one of the self-linking elements in its second symbolic power. Finally, in Section 4, we'll study what it means for the variety to be normal.

A milestone in this theory was reached by Catanese [6] on the basis of work by and with a number of others. Catanese (see [6, Rmk. 5.1, p. 98]) set up a correspondence between Gorenstein algebras with module generators and symmetric matrices satisfying a rank condition, or row condition, (RC); here each algebra is birational onto its image, the generators form a finite minimal set starting with 1 , and $\mathrm{RC}$ means that the ideal of submaximal minors of the matrix is equal to the ideal of maximal minors of the submatrix obtained by deleting the first row. Viewing $\mathrm{RC}$ as the hypothesis of the Rouché-Capelli theorem (which asserts that an inhomogeneous system of linear equations has a solution if the rank of the coefficient matrix is equal to that of its augmentation), Catanese [6, Rmk. 4.6, p. 91] showed that $\mathrm{RC}$ is essentially equivalent to the existence of a commutative associative algebra structure on the cokernel of the matrix. Catanese also considered algebras of higher rank, especially of rank two, and represented them via symmetric matrices; however, $\mathrm{RC}$ is no longer relevant. (In fact, Catanese considered weighted homogeneous algebras, but the theory is essentially the same for our purposes.) Reviewing Catanese's paper, Reid [MR 86c:14027] called the correspondence "the most interesting idea of this important paper."

The correspondence was extended by Mond and Pellikaan [26] and by de Jong and van Straten [7]. Mond and Pellikaan were especially interested in the locus $N_{i}$, for $i=1,2,3, \ldots$, defined by the vanishing of the $(i-1)$-st Fitting ideal of the algebra. Their purpose was to show that these loci are suitable choices for the loci of multiple points of the projection. For example, $N_{1}$ is the image of the projection; $N_{2}$ is the locus where the projection is not an isomorphism, with the structure defined by the adjoint ideal; and $N_{3}$ is, in the Gorenstein case, a particularly wellbehaved locus of triple points. De Jong and van Straten were especially interested in relating the deformation theory of the projection to the deformation theory of the inclusion of $N_{2}$ into $N_{1}$. In particular, they often worked over a nonreduced base ring containing the complex numbers.

Mond and Pellikaan and de Jong and van Straten extended the correspondence to relate Cohen-Macaulay algebras with generators and square matrices with RC. Furthermore, they added a third component, the Cohen-Macaulay ideals $I$ of codimension 2 with a preferred element $\Delta$ satisfying a ring condition; this is the condition that the reciprocal of $I /(\Delta)$, viewed as a fractional ideal, is equal to the endomorphism ring of $I /(\Delta)$. The present authors, together with Lipman, developed the connection with enumerative multiple-point theory in [21] and [22]. In the present paper, we will investigate the third component of the correspondence, especially in the Gorenstein case in part using the symbolic Rees algebra, and we'll study the significance of normality. Along the way, we'll extend some of the known results, and simplify and clarify their proofs. In more detail, here's what we'll do.

We will work, for the most part, over an arbitrary Noetherian local ring $R$, which plays the role of the localized polynomial ring. In this setting, the correspondence relates $R$-algebras $B$ with module generators, square matrices $\varphi$ with RC, and ideals $I$ with a preferred element $\Delta$ satisfying the ring condition. Moreover, we'll replace the condition that $R$ be regular and $B$ (resp., $I$ ) be Cohen-Macaulay with the more general condition that $B$ be perfect of grade 1 (resp., that $I$ be perfect of grade 
2). Similarly, we'll use the relative form of the Gorenstein condition, namely, that $\operatorname{Ext}_{R}^{1}(B, R)$ be $B$-isomorphic to $B$.

In Section 2, we will prove the symmetrization results. First, Theorem 2.3 asserts that, if a Gorenstein $R$-algebra $B$ is presented by a matrix $\varphi$ with regular determinant, then there exist invertible matrices $\varepsilon$ and $\mu$ such that $\varepsilon \varphi$ and $\varphi \mu$ are symmetric. Conversely, an $R$-algebra $B$ is Gorenstein if it's presented by a matrix with regular determinant $\Delta$ and if $R /(\Delta) \subset B$; see Proposition 2.12. Second, Theorem 2.6 asserts that a necessary and sufficient condition for a perfect $R$-ideal $I$ of grade two to be self-linked is that, given any $n$ by $n-1$ matrix $\phi$ presenting $I$, there exists an invertible matrix $\varepsilon$ such that the submatrix consisting of the last $n-1$ rows of $\varepsilon \phi$ is symmetric. Our proofs of Theorems 2.3 and 2.6 are substantially easier, shorter, and more general than earlier proofs; our proofs are our main contribution in Section 2.

Theorem 2.3 and the necessity in Theorem 2.6 are derived from Lemma 2.1, which shifts the burden of proving the symmetry of a matrix to checking the condition that the syzygy matrix of the matrix modulo a nonzerodivisor is symmetric, which is a natural condition in the context of the two theorems. Lemma 2.1 enters via Proposition 2.2, which adds another common ingredient, a bilinear form. This line of reasoning was inspired by Catanese's original argument [6, pp. 84-87]. Theorem 2.3 was proved independently and in essentially the same way by Grassi [15], and he went on to treat perfect Gorenstein algebras of grade 2. Another proof of Theorem 2.3 was given by Mond and Pellikaan [26, Prop. 2.5, p. 117], but their proof uses Noether normalization, and breaks down when $B$ is not finite over a regular subring.

The sufficiency assertion of Theorem 2.6 is derived in a few lines from Part (3) of Lemma 2.5, which asserts this: given a symmetric matrix whose ideal of submaximal minors that do not involve the first column is of grade at least 2, the determinant and the lower right minor together form a regular sequence with respect to which the ideal is self-linked. This assertion also follows from a theorem of Valla's [32, Thm. 2.1, p. 97]; Valla's proof uses the mapping cone, whereas ours uses matrix factorization. The use of matrix factorization in this context was introduced by de Jong and van Straten [7, p. 532], who used it to prove a version of Parts (1) and (2) of Lemma 2.5; see also [26, pf. of 3.14, pp. 126-7]. Theorem 2.6 was first stated and proved in full by Ferrand in an unpublished fifteen-page manuscript, but his proof of necessity, unlike ours, requires that 2 be invertible in $R$. Valla [32, Remark, p. 99] posed the problem of finding an easier proof of necessity, and then gave one in the case of three generators. Ours is such a proof, which works for arbitrarily many generators and in arbitrary characteristic.

Proposition 2.9 gives, for a perfect $R$-algebra $B$ of grade 1 , an upper bound on the height of its Fitting ideal $F_{i}$ for $i \geq 2$. In geometric terms, this is an upper bound on the codimension of the multiple-point locus $N_{i+1}$ of a central projection. Part (1) of the proposition asserts that the height of $F_{i}$ is at most $\left(\begin{array}{c}i+1 \\ 2\end{array}\right)$ if $B$ is Gorenstein. Part (2) asserts that the height of $F_{2}$ is at most 4 if $B$ is CohenMacaulay and is of rank 2 over $R /(\Delta)$, where $\Delta$ is a nonzerodivisor, and if $R$ is regular. Part (1) for $i=2$ was proved (implicitly) by Mond and Pellikaan [26, Thm. 4.3, p. 131] when $B$ is of rank 1 over its image (or birational onto it). In particular, in the Gorenstein case, the codimension of $N_{3}$ is at most 3, which is the expected upper bound. However, this bound can fail in the Cohen-Macaulay case; 
see Example 2.10. It is unknown whether it is possible to have $\operatorname{cod} N_{4}>4$ in the Gorenstein case.

Both parts of Proposition 2.9 will be derived from Lemma 2.8, which asserts that $F_{i}$ has the same radical as a certain subideal, the subideal of $n-i$ by $n-i$ minors of the matrix obtained by deleting the first row and column of a suitable matrix presenting $B$. In Part (1) the matrix is symmetric, and in Part (2) it's alternating. In their case, Mond and Pellikaan [26, Prop. 4.1, p. 128] proved that the two ideals coincide. We'll recover this result, via their approach, as part of Lemma 3.6. In Part (2) the matrix is given by Proposition 2.7(1), which deals more generally with modules $M$ of rank 2 over $R /(\Delta)$; the matrix is obtained directly from Proposition 2.2. Proposition 2.7(2) recovers a result of Herzog and Kühl [17, Thm. 3.1(a), p. 82], which asserts that the minimal number of generators $\nu(M)$ is even. They follow a different approach: they associate a Gorenstein ideal of grade 3 to $M$ via a Bourbaki sequence, and then use the Buchsbaum-Eisenbud structure theorem, which says that these ideals are presented by an alternating matrix. Proposition 2.7(3) gives an upper bound on the height of $\operatorname{Fitt}_{i}(M)$ for $i<\nu(M) / 2$; the special case $i=1$ is also a special case of a result of Bruns [3, Cor. 2, p. 23].

In Section 3, we'll establish the correspondence between the set of perfect $R$ algebras $B$ of grade 1 whose first and second Fitting ideals $F_{1}$ and $F_{2}$ satisfy grade $F_{1}=2$ and grade $F_{2} \geq 3$ and the set of the perfect $R$-ideals $I$ of grade 2 such that $I$ is a complete intersection at each associated prime and whose second symbolic power $I^{(2)}$ contains a preferred regular element $\Delta$, determined up to a unit multiple. The condition grade $F_{1}=2$ means simply that $B$ lies in the total quotient ring of $R /(\Delta)$ and that $B \neq R /(\Delta)$; see Proposition 2.12. The Gorenstein $B$ correspond to the $I$ that are self-linked with $\Delta$ as one of the self-linking elements. Given $B$, we'll take $I$ to be $F_{1}$, and $\Delta$ to be a generator of $F_{0}$. Given $I$ and $\Delta$, we'll take $B$ to be the reciprocal of $I /(\Delta)$ in the total ring of quotients of $R /(\Delta)$. Proposition 3.1 starts with an $I$ and $\Delta$; it asserts that the corresponding $B$ has the desired properties - in particular, that $B$ is a ring whose first Fitting ideal $F_{1}$ is equal to $I$, and that $B$ is Gorenstein if and only if $I=(\Delta, \alpha): I$ for some $\alpha$. The proof shows that $B$ is presented by a square matrix with RC. In fact, its transpose presents $I /(\Delta)$ and the transpose of its truncation is a Hilbert-Burch matrix of $I$. Hence $F_{1}$ is equal to the first Fitting ideal of $I /(\Delta)$, and RC means simply that $F_{1}=I$. Conversely, Proposition 3.6 starts with an algebra $B$ and asserts that its first Fitting ideal has most of the desired properties; the remaining ones follow from Proposition 3.1. The correspondence is summarized formally in Theorem 3.7. These results extend the corresponding work of Mond and Pellikaan [26] and of de Jong and van Straten [7], with the notable exception of the characterization of the Gorenstein $B$, which is new.

Our version of the correspondence features two conditions: (i) that $\Delta \in I^{(2)}$, and (ii) that $I$ is self-linked with $\Delta$ as one of the self-linking elements. In the geometric case, under the assumption that $R / I$ is reduced, the equivalence of (i) with the ring condition was explicitly proved by de Jong and van Straten [7, Thm. 1.2, p. 529]. In this situation, $I$ is generically a complete intersection; so their case is covered by ours. Moreover, in their case, the map $d: I / I^{2} \rightarrow \Omega_{R}^{1} \otimes R / I$ is generically injective; hence, (i) means, in geometric terms, that the hypersurface defined by the vanishing of $\Delta$ is singular along the zero locus of $I$. On a different tack, Eisenbud and Mazur, inspired by the recent Taylor-Wiles proof of Fermat's 
last theorem, proved the following higher-codimensional version of the equivalence of (i) and RC [10, Thm. 4]: if $I$ has no embedded primes and has grade at least $r$, then (i) implies that the $(r-1)$-st Fitting ideal of $I /(\Delta)$ is contained in $I$, and the converse holds if also $I$ is a complete intersection of height $r$ locally at each associated prime. As to (ii), it is equivalent to the condition that $B$ is Gorenstein, given that $B$ is a ring. Indeed, it is not hard to see that $\alpha$ is simply an adjoint; that is, $\alpha B=I /(\Delta)$. Nevertheless, this equivalence seems not to have been observed before.

In addition to having intrinsic interest, these conditions (i) and (ii) suggest considering the symbolic Rees algebra $\mathcal{R}_{s}(I):=\bigoplus_{n \geq 0} I^{(n)} t^{n}$. Indeed, pursuing the work of a number of others, Herzog and Ulrich [18, Cor. 2.5, p. 146] proved that, if $\mathcal{R}_{s} I=R\left[I t, I^{(2)} t^{2}\right]$, then $I$ is self-linked in the case that $R$ is regular of dimension 3 , and that $I$ is of codimension 2 and satisfies certain technical conditions. We will, in a way, generalize this result, but our results are really new.

Theorem 3.4 asserts that, if $R$ is Cohen-Macaulay with infinite residue class field and if $I$ is perfect of grade 2 and is a complete intersection at each associated prime, then a necessary and sufficient condition for $I$ to be self-linked with one of the self-linking elements in $I^{(2)}$ is that $\mathcal{R}_{s} I=R\left[I t, \Delta t^{2}\right]$ for some $\Delta \in I^{(2)}$ and that $I^{(i)}$ be perfect for every $i>0$. In fact, necessity holds without the CohenMacaulay hypothesis, see Proposition 3.3, and we suspect that sufficiency does too. Furthermore, if $I$ is self-linked with respect to some $\Delta \in I^{(2)}$ and $\alpha \in I$, then any general $\Delta$ and $\alpha$ will do; this is an unusual phenomenon, and means that, in examples, a random choice of $\Delta$ and $\alpha$ will likely do. Theorem 3.4 also gives another necessary and sufficient condition, namely, that $I^{(2)} / I^{2}$ be cyclic and the analytic spread $\ell\left(I^{(2)}\right)$ be 2 . Moreover, if the equivalent conditions of Theorem 3.4 hold then, curiously, all the symbolic powers $I^{(i)}$ are self-linked. In addition, then, by Proposition 3.5, the symbolic Rees algebra $\mathcal{R}_{s}(I)$ is Cohen-Macaulay, and it is Gorenstein if $R$ is.

The quotient $I^{(2)} / I^{2}$ is rather interesting. Indeed, Proposition 3.8 asserts that, if $B$ is Gorenstein, then $\Delta$ defines an isomorphism, $R / F_{2} \stackrel{\sim}{\longrightarrow} I^{(2)} / I^{2}$. This result was proved under the additional hypothesis that $F_{1}$ is radical by Mond and Pellikaan [26, Thm. 4.4, p. 132]. In addition, they pointed out its "somewhat surprising" geometric significance: the triple-point locus of a projection depends only on the double-point locus, provided these loci have at least the expected codimensions. Another proof was given by de Jong and van Straten [7, Thm. 2.8, p. 538].

In Section 4, we'll return to the correspondence between the algebra $B$ and the pair $(I, \Delta)$. It seems natural to seek critera guaranteeing that $B$ is the entire integral closure of $A:=R /(\Delta)$. Assume that $R$ satisfies Serre's conditions $\left(\mathrm{R}_{2}\right)$ and $\left(\mathrm{S}_{3}\right)$. Proposition 4.1 asserts notably that, if $B$ is normal, then $\Delta \in I^{(2)}$ if and only if $A:=R /(\Delta)$ has multiplicity 2 locally at each associated prime of $I$. Consequently, it is easy to find an example in which $B$ is a normal ring, yet $\Delta \notin I^{(2)}$; see Example 4.2. Finally, Theorem 4.4 asserts notably that, if $R$ contains an infinite and perfect field, if $I$ is a complete intersection locally at each associated prime, and if $\Delta$ is a general element of $I^{(2)}$, then $B$ is normal if and only if $A$ has multiplicity 2 locally at each associated prime of $I$; furthermore, if so, then $B / A$ is unramified in codimension 1. In other words, if $\Delta \in I^{(2)}$ is general, then the hypersurface it defines is the image of the projection of a normal variety with $I$ as its adjoint ideal if and only if the hypersurface, generically along the zeros of $I$, 
consists of two smooth sheets, which may be tangent. Example 4.5 shows that it is necessary that $\Delta$ be taken general.

\section{Symmetric matrices}

In this section, we prove that, if a Gorenstein algebra $B$ can be presented by a square matrix $\varphi$ with regular determinant, then we can take $\varphi$ to be symmetric; in fact, we can make $\varphi$ symmetric via row operations, or if we prefer via column operations. We use this result to derive a bound on the heights of the Fitting ideals of $B$. We also prove that a perfect ideal $I$ of grade 2 is self-linked if and only if, via row operations, we can make any given $n$ by $n-1$ matrix presenting $I$ possess a symmetric $n-1$ by $n-1$ subblock.

Lemma 2.1. Let $(R, m)$ be a local ring, and let lower-case Greek letters stand for $n$ by $n$ matrices with entries in $R$. Let $\varphi$ be such a matrix, and let $r$ denote its rank modulo $m$. Let $\Delta$ be a nonzerodivisor in $R$ contained in the annihilator of $\operatorname{Cok} \varphi$, and let "-, indicate reduction modulo $\Delta$. Further, assume that there is an exact sequence,

$$
\bar{R}^{n} \stackrel{\bar{\psi}}{\rightarrow} \bar{R}^{n} \stackrel{\bar{\varphi}}{\rightarrow} \bar{R}^{n},
$$

where $\bar{\psi}$ is either symmetric or alternating; in the latter case, assume that $r$ is even. Then there exist invertible matrices $\varepsilon$ and $\mu$ such that the products $\varepsilon \varphi$ and $\varphi \mu$ are symmetric or alternating, respectively.

Proof. It suffices to show that there exist invertible matrices $\alpha, \beta$ such that $\alpha \varphi \beta$ is symmetric (or alternating). Indeed, let ${ }^{*}$ ' indicate transpose, and set $\varepsilon:=$ $\beta^{-1 *} \alpha$ and $\mu:=\beta \alpha^{-1 *}$. Then $\varepsilon \varphi$ is equal to $\beta^{-1 *} \alpha \varphi \beta \beta^{-1}$, which is obviously then symmetric (or alternating). Similarly, $\varphi \mu$ is symmetric (or alternating).

Because $(R, m)$ is local, there exist invertible matrices $\alpha, \beta$ such that $\alpha \varphi \beta$ has the form,

$$
\left(\begin{array}{cc}
\mathbf{1}_{r \times r} & \mathbf{0} \\
\mathbf{0} & \varphi^{\prime}
\end{array}\right)
$$

or, if $r=2 s$, the form,

$$
\left(\begin{array}{ccc}
\mathbf{0} & \mathbf{1}_{s \times s} & \mathbf{0} \\
-\mathbf{1}_{s \times s} & \mathbf{0} & \mathbf{0} \\
\mathbf{0} & \mathbf{0} & \varphi^{\prime}
\end{array}\right),
$$

where the entries of $\varphi^{\prime}$ are in $m$. Replacing $\varphi$ by $\alpha \varphi \beta$ and $\psi$ by $\beta^{-1} \psi \beta^{-1 *}$, we may assume that $\varphi$ itself has the displayed form. Now, $\bar{\psi} \bar{\varphi}=\mathbf{0}$, and $\bar{\psi}$ is symmetric (or alternating). Hence, we may assume that $\bar{\psi}$ has the form,

$$
\left(\begin{array}{cc}
\overline{\mathbf{0}}_{r \times r} & \overline{\mathbf{0}} \\
\overline{\mathbf{0}} & \bar{\psi}^{\prime}
\end{array}\right)
$$

where $\bar{\psi}^{\prime}$ is still symmetric (or alternating). Therefore, the following sequence is exact:

$$
\bar{R}^{n-r} \stackrel{\bar{\psi}^{\prime}}{\rightarrow} \bar{R}^{n-r} \stackrel{\bar{\varphi}^{\prime}}{\rightarrow} \bar{R}^{n-r}
$$

So, replacing $\varphi$ by $\varphi^{\prime}$, we may assume that all the entries of $\varphi$ lie in $m$.

We may further assume that $\psi$ is symmetric (or alternating). Using the exact sequence (2.1.1), we now prove (in a manner reminiscent of an argument used by 
Shamash [30, pf. of Lem. 1, p. 454]) and by Eisenbud [9, 6.3, p. 55]) that the inverse matrix $\psi^{-1}$ is defined over the total quotient ring $K$ of $R$ and that there exists an invertible matrix $\varepsilon$ such that $\varepsilon \varphi=\Delta \psi^{-1}$.

First of all, $\Delta \cdot \mathbf{1}_{n \times n}=\varphi \chi$ for some $\chi$; indeed, since $\Delta$ annihilates the cokernel of $\varphi$, every column of $\Delta \cdot \mathbf{1}_{n \times n}$ is a relation on $\operatorname{Cok} \varphi$, and so lies in the column space of $\varphi$. Since $\Delta$ is a nonzerodivisor, $\varphi^{-1}$ and $\chi^{-1}$ exist over $K$ and

$$
\chi=\Delta \varphi^{-1} \text {. }
$$

Consequently,

$$
\chi \varphi=\varphi \chi=\Delta \cdot \mathbf{1}_{n \times n} .
$$

Since $\overline{\varphi \chi}$ vanishes and since (2.1.1) is exact, there exists a $\gamma$ such that $\bar{\chi}=\bar{\psi} \bar{\gamma}$. Hence, for some $\lambda$,

$$
\chi=\psi \gamma+\Delta \lambda=\psi \gamma+\chi \varphi \lambda,
$$

where the second equality follows from (2.1.3). Now, the entries of $\varphi$ lie in $m$; hence, $1-\varphi \lambda$ is invertible. Set $\varepsilon:=\gamma(1-\varphi \lambda)^{-1}$. Then

$$
\chi=\psi \varepsilon .
$$

Hence, since $\chi$ is invertible over $K$, so are $\psi$ and $\varepsilon$.

On the other hand, since (2.1.1) is a complex, there exists some $\eta$ such that

$$
\varphi \psi=\Delta \eta=\varphi \chi \eta \text {. }
$$

Multiplication by $\varphi^{-1}$ now yields $\psi=\chi \eta$. Substituting into (2.1.4), we conclude that $\varepsilon$ is, in fact, invertible over $R$. Finally, (2.1.4) and (2.1.2) yield that $\varepsilon \varphi=$ $\Delta \psi^{-1}$. Now, $\psi^{-1}$ is symmetric (or alternating). Hence $\alpha:=\varepsilon$ and $\beta:=\mathbf{1}_{n \times n}$ are as required, and the proof is complete.

Proposition 2.2. Let $(R, m)$ be a local ring. Let $\Delta$ be a nonzerodivisor in $R$, and set $A:=R /(\Delta)$. Let $M$ be an $A$-module that is presented, as an $R$-module, by an $n$ by $n$ matrix $\varphi$. Assume that there exists a bilinear form $b: M \times M \rightarrow A$ which induces a surjection,

$$
M \rightarrow \operatorname{Hom}_{A}(M, A),
$$

and which is either symmetric or alternating. In the latter case, assume that

$$
n \equiv \nu(M) \bmod 2,
$$

where $\nu(M)$ denotes the minimal number of generators of $M$. Then there are invertible $n$ by $n$ matrices $\varepsilon$ and $\mu$ such that the products $\varepsilon \varphi$ and $\varphi \mu$ are symmetric or alternating, respectively.

Proof. Let $u_{1}, \ldots, u_{n}$ be generators of $M$ corresponding to the presentation $\varphi$. Let " $\rightarrow$ indicate images in $A$, and let "*) indicate the dual of a given $A$-module or $A$-map. Now, dualizing the exact sequence,

$$
A^{n} \stackrel{\bar{\varphi}}{\rightarrow} A^{n} \rightarrow M \rightarrow 0
$$

yields an exact sequence,

$$
0 \rightarrow M^{*} \rightarrow A^{n *} \stackrel{\bar{\varphi}^{*}}{\longrightarrow} A^{n *} .
$$


Set $\bar{\psi}:=\left(b\left(u_{i}, u_{j}\right)\right)$. This is a symmetric (or alternating) $n$ by $n$ matrix with entries in $A$. Since the $u_{i}$ correspond to the presentation of $M$ by $\varphi$, our assumption on $b$ and the exactness of (2.2.1) imply the exactness of the following sequence:

$$
A^{n} \stackrel{\bar{\psi}}{\rightarrow} A^{n *} \stackrel{\bar{\varphi}^{*}}{\rightarrow} A^{n *}
$$

Obviously, modulo $m$, the rank of $\varphi$ is equal to $n-\nu(M)$. Hence, Lemma 2.1 yields the assertion.

Theorem 2.3. Let $R$ be a Noetherian local ring, $B$ a Gorenstein $R$-algebra. If $B$ is presented, as an $R$-module, by an $n$ by $n$ matrix $\varphi$ with regular determinant, then there exist invertible $n$ by $n$ matrices $\varepsilon$ and $\mu$ such that $\varepsilon \varphi$ and $\varphi \mu$ are symmetric.

Proof. Set $\Delta:=\operatorname{det} \varphi$ and $A:=R /(\Delta)$. Since $\Delta$ is $R$-regular and kills $B$, the sequence $0 \rightarrow R \stackrel{\Delta}{\rightarrow} R \rightarrow A \rightarrow 0$ is exact and yields a natural isomorphism of $B$-modules, $\operatorname{Hom}_{A}(B, A)=\operatorname{Ext}_{R}^{1}(B, R)$. This Ext is $B$-isomorphic to $B$ since $B$ is Gorenstein over $R$. Therefore, for some $t \in \operatorname{Hom}_{A}(B, A)$,

$$
\operatorname{Hom}_{A}(B, A)=B t \text {. }
$$

Let $m: B \times B \rightarrow B$ be multiplication on the commutative ring $B$, and consider the symmetric $A$-valued bilinear form $b:=t \circ m$. The equality (2.3.1) implies that $b$ induces a surjection,

$$
B \rightarrow \operatorname{Hom}_{A}(B, A) .
$$

Hence, the assertion follows from Proposition (2.2).

Lemma (Laplace identity) 2.4. Let $R$ be a commutative ring, $\varphi$ an $n$ by $n$ matrix with entries in $R$. Set $\Delta:=\operatorname{det} \varphi$, denote by $m_{i}^{j}$ the minor of $\varphi$ obtained by deleting Row $i$ and Column $j$, and denote by $m_{i, k}^{j, l}$ that obtained by deleting Rows $i$ and $k$ and Columns $j$ and $l$, where $m_{i, k}^{j, l}=0$ if $i=k$ or $j=l$, and where the determinant of the empty matrix is 1 . Then, for $1 \leq i \leq k \leq n$ and $1 \leq j \leq l \leq n$,

$$
m_{i, k}^{j, l} \Delta=m_{i}^{j} m_{k}^{l}-m_{i}^{l} m_{k}^{j} .
$$

Proof. We may assume that $1=i<k$ and $1=j<l$. Let $\phi^{\prime}$ be the $n-1$ by $n-1$ matrix obtained by deleting the first row and column of $\varphi$, and let $\theta$ be the $n$ by $n$ matrix obtained from $\operatorname{adj}\left(\phi^{\prime}\right)$ by adding a row of 0 s at the top and a column of 0 s on the left. We can now check the asserted identity by multiplying the familiar equation,

on the left by $\theta$.

$$
\Delta \mathbf{1}_{n \times n}=\varphi \operatorname{adj}(\varphi)
$$

Lemma 2.5. Let $R$ be a Noetherian local ring, $\varphi$ an $n$ by $n$ matrix with entries in $R$. Let $\phi$ be the $n$ by $n-1$ matrix consisting of the last $n-1$ columns of $\varphi$, and $\phi^{\prime}$ the submatrix of $\phi$ consisting of the last $n-1$ rows. Set $\Delta:=\operatorname{det} \varphi$, set $\alpha:=\operatorname{det} \phi^{\prime}$, and set $I:=\mathbf{I}_{n-1}(\phi)$, the ideal of $n-1$ by $n-1$ minors. Assume grade $I \geq 2$. Set $A:=R /(\Delta)$ and let ${ }^{-}{ }^{-}$, indicate the image in $A$.

(1) Let ${ }^{\prime *}$ ' indicate the transpose. If $\Delta$ is regular, then

$$
\begin{gathered}
\bar{I}=\operatorname{Cok}(\bar{\varphi})=\operatorname{Im}(\operatorname{adj} \bar{\varphi})=\operatorname{Ker}(\bar{\varphi}), \\
\operatorname{Hom}_{A}(\bar{I}, A)=\operatorname{Ker}\left(\bar{\varphi}^{*}\right)=\operatorname{Im}\left(\operatorname{adj} \bar{\varphi}^{*}\right)=\operatorname{Cok}\left(\bar{\varphi}^{*}\right) .
\end{gathered}
$$


(2) If $\Delta$ is regular, then

$$
\mathbf{I}_{n-1}(\varphi)=\mathbf{I}_{n-1}(\phi) \text { if and only if } \operatorname{Hom}_{A}(\bar{I}, A)=\operatorname{Hom}_{A}(\bar{I}, \bar{I}) .
$$

(3) If $\varphi$ is symmetric, then $\Delta, \alpha$ form an $R$-regular sequence, and $I$ is self-linked with respect to $\Delta, \alpha$; that is, $(\Delta, \alpha): I=I$.

Proof. Let $\Delta_{1}, \ldots, \Delta_{n}$ be the signed maximal minors of $\phi$, and say

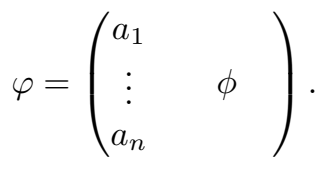

Then $\Delta=\sum a_{i} \Delta_{i}$. Since grade $I \geq 2$, the Hilbert-Burch sequence,

$$
R^{n-1} \stackrel{\phi}{\rightarrow} R^{n} \rightarrow I \rightarrow 0
$$

is exact. Hence, it induces this exact sequence:

$$
A^{n} \stackrel{\bar{\varphi}}{\rightarrow} A^{n} \rightarrow \bar{I} \rightarrow 0 .
$$

Dualizing the latter yields the following exact sequence:

$$
0 \rightarrow \operatorname{Hom}_{A}(\bar{I}, A) \rightarrow A^{n *} \stackrel{\bar{\varphi}^{*}}{\rightarrow} A^{n *} .
$$

On the other hand, it is not hard to show that, if $\Delta$ is regular, then the matrix factorization $\varphi \cdot \operatorname{adj} \varphi=\Delta \mathbf{1}_{n \times n}$ yields the following two periodic exact sequences (see [9, Prop. 5.1, p. 49]):

$$
\begin{gathered}
\cdots \rightarrow A^{n} \stackrel{\bar{\varphi}}{\rightarrow} A^{n} \stackrel{\text { adj } \bar{\varphi}}{\longrightarrow} A^{n} \stackrel{\bar{\varphi}}{\rightarrow} A^{n} \rightarrow \cdots ; \\
\cdots \rightarrow A^{n *} \stackrel{\bar{\varphi}^{*}}{\rightarrow} A^{n *} \stackrel{\text { adj } \bar{\varphi}^{*}}{\longrightarrow} A^{n *} \stackrel{\bar{\varphi}^{*}}{\rightarrow} A^{n *} \rightarrow \cdots .
\end{gathered}
$$

Therefore, (1) holds.

Assume that $\Delta$ is regular. Let $u_{i}: \bar{I} \rightarrow A$, where $1 \leq i \leq n$, be the generators of $\operatorname{Hom}_{A}(\bar{I}, A)$ that arise, in the above presentation of this module, from the dual basis of $A^{n *}$. Then

$$
\operatorname{adj} \bar{\varphi}^{*}=\left(u_{i}\left(\bar{\Delta}_{j}\right)\right)
$$

Thus $\mathbf{I}_{n-1}(\bar{\varphi})=\bar{I}$ if and only if $u_{i}(\bar{I}) \subset \bar{I}$ for every $i$. It follows that (2) holds.

Assertion (3) holds by virtue of some of Valla's work; see [32, Thm. 2.1, p. 97]. However, the following proof is more in the spirit of the present paper. Use the notation of the preceding lemma. Then $m_{1}^{1}=\alpha$ and $m_{i}^{1}=(-1)^{i+1} \Delta_{i}$. So the preceding lemma (the Laplace identity) yields

$$
m_{1, k}^{j, 1} \Delta=m_{1}^{j} m_{k}^{1}-\alpha m_{k}^{j} .
$$

Assume $\varphi$ is symmetric. Then $m_{1}^{j}=m_{j}^{1}$. Hence $(\Delta, \alpha) \supset I^{2}$. Consequently, $\Delta, \alpha$ form an $R$-regular sequence.

Let $K$ denote the total ring of quotients of $A$. Then $\bar{I} K=K$ because $\bar{I}$ contains a nonzerodivisor, namely, $\bar{\alpha}$. View $\operatorname{Hom}_{A}(\bar{I}, A)$ as a submodule of $\operatorname{Hom}_{K}(\bar{I} K, K)$, and view the latter as $K$ by identifying a map $u$ with multiplication by $u(1)$. Then $\operatorname{Hom}_{A}(\bar{I}, A)$ becomes identified with $A:_{K} \bar{I}$, and $\operatorname{Hom}_{A}(\bar{I}, \bar{I})$ with $\bar{I}:_{K} \bar{I}$. Consider the image of $\operatorname{Hom}_{A}(\bar{I}, A)$ in $A^{n *}$, and project it on the first coordinate; clearly, the projection is $\left(A:_{K} \bar{I}\right) \bar{\alpha}$. The latter is equal to $(\bar{\alpha}):_{K} \bar{I}$, so to $(\bar{\alpha}):_{A} \bar{I}$ because $\bar{\alpha} \in \bar{I}$ is a nonzerodivisor. On the other hand, the image of $\operatorname{Hom}_{A}(\bar{I}, A)$ is equal 
to $\operatorname{Im}\left(\operatorname{adj} \bar{\varphi}^{*}\right)$ by $(1)$. Hence its projection is equal to the ideal generated by the first row of adj $\bar{\varphi}^{*}$. Since $\varphi$ is symmetric, the latter is $\left(\bar{\Delta}_{1}, \ldots, \bar{\Delta}_{n}\right)$, or $\bar{I}$. Thus $(\bar{\alpha}): \bar{I}=\bar{I}$, and so $(\Delta, \alpha): I=I$. The proof is now complete.

Theorem 2.6. Let $R$ be a Noetherian local ring, let $I$ be a perfect $R$-ideal of grade two, and let $\phi$ be an $n$ by $n-1$ matrix with entries in $R$ presenting $I$. Then the following two conditions are equivalent:

(i) The ideal I is self-linked.

(ii) There exists an invertible $n$ by $n$ matrix $\varepsilon$ with entries in $R$ such that the $n-1$ by $n-1$ matrix consisting of the last $n-1$ rows of $\varepsilon \phi$ is symmetric.

Proof. We first show that (i) implies (ii). Assume that $(\Delta, \alpha): I=I$. Then $\Delta, \alpha$ form an $R$-regular sequence contained in $I$, because

$$
I^{2} \subset(\Delta, \alpha) \subset(\Delta, \alpha): I=I .
$$

Let $\Delta_{1}, \ldots, \Delta_{n}$ be the signed maximal minors of $\phi$. They generate $I$ by the Hilbert-Burch Theorem, which applies because $I$ is perfect of grade two. So, since $\Delta$ is in $I$, there are elements $a_{1}, \ldots, a_{n}$ of $R$ such that $\Delta=\sum a_{i} \Delta_{i}$. Set

$$
\varphi:=\left(\begin{array}{cc}
a_{1} & \\
\vdots & \phi \\
a_{n} &
\end{array}\right) .
$$

Then $\operatorname{det} \varphi=\Delta$. Set $A:=R /(\Delta)$ and let ' ${ }^{-}$' indicate the image in $A$. Notice that the following sequence is exact:

$$
R^{n} \stackrel{\varphi}{\rightarrow} R^{n} \rightarrow \bar{I} \rightarrow 0
$$

Let $K$ denote the total ring of quotients of $A$, and $t: K \rightarrow K$ multiplication by $1 / \bar{\alpha}$. As in the proof of Lemma 2.5, we can see that $\operatorname{Hom}_{A}(\bar{I}, A)$ is equal to $A:_{K} \bar{I}$. The latter is clearly equal to $\left(\bar{\alpha}:_{A} \bar{I}\right)(1 / \bar{\alpha})$. Hence, the self-linkage assumption yields

$$
\operatorname{Hom}_{A}(\bar{I}, A)=\bar{I} t .
$$

On the other hand, since $\bar{I}^{2} \subset(\bar{\alpha})$, multiplication on $A$, followed by $t$, defines a symmetric bilinear form $b: \bar{I} \times \bar{I} \rightarrow A$. By virtue of (2.6.1), $b$ induces an isomorphism,

$$
\bar{I} \stackrel{\sim}{\longrightarrow} \operatorname{Hom}_{A}(\bar{I}, A) .
$$

Proposition 2.2 now implies (ii).

Conversely, assume (ii). Since $\varepsilon$ is invertible, $\varepsilon \phi$ also presents $I$. Hence, we may assume that

$$
\phi=\left(\begin{array}{ccc}
a_{2} & \cdots & a_{n} \\
& \phi^{\prime} &
\end{array}\right)
$$

for suitable elements $a_{2}, \ldots, a_{n}$ and a suitable symmetric $n-1$ by $n-1$ matrix $\phi^{\prime}$. Set

$$
\varphi:=\left(\begin{array}{cccc}
0 & a_{2} & \cdots & a_{n} \\
a_{2} & & & \\
\vdots & & \phi^{\prime} & \\
a_{n} & & &
\end{array}\right)
$$

and apply Lemma 2.5. Thus (ii) implies (i), and the proof is complete. 
Proposition 2.7. Let $R$ be a Noetherian local ring, $\Delta$ an $R$-regular element, and set $A:=R /(\Delta)$. Let $M$ be an A-module. Assume that $M_{p}$ is a free $A_{p}$-module of rank two for every prime ideal $p$ of $A$ with $\operatorname{depth} A_{p} \leq 1$, that $M$ is orientable (that is, $\left(\wedge^{2} M\right)^{* *} \cong A$ where ${ }^{*}$, indicates the dual module), and that $M$ is presented, as an $R$-module, by an $n$ by $n$ matrix $\varphi$ with $n \equiv \nu(M) \bmod 2$, where $\nu(M)$ denotes the minimal number of generators of $M$. (For instance, let $M$ be an orientable maximal Cohen-Macaulay module of rank two over a hypersurface ring.)

(1) Then there are invertible $n$ by $n$ matrices $\varepsilon$ and $\mu$ with entries in $R$ such that $\varepsilon \varphi$ and $\varphi \mu$ are alternating.

(2) (Herzog and Kühl [17, Thm. 3.1(a), p. 82]) Then $\nu(M)$ is even.

(3) For $i<\nu(M) / 2$,

$$
\text { height } \operatorname{Fitt}_{2 i}^{A}(M)=\text { height } \operatorname{Fitt}_{2 i+1}^{A}(M) \leq 2 i^{2}+3 i .
$$

Proof. To prove (1), consider the alternating bilinear form,

$$
b: M \times M \rightarrow \wedge^{2} M \rightarrow\left(\wedge^{2} M\right)^{* *} \stackrel{\sim}{\longrightarrow} A,
$$

where the first two maps are the natural maps; it induces a surjection $M \rightarrow M^{*}$ since $M$ is free locally in depth 1 . Hence Proposition 2.2 yields (1).

For (2), we may assume that $n=\nu(M)$ and that $\varphi$ is alternating. Notice that

$$
\Delta \in \operatorname{Ann}_{R}(M) \subset \sqrt{\operatorname{Fitt}_{0}^{R}(M)} .
$$

In particular, $\operatorname{det} \varphi \neq 0$, and hence $n$ is even.

Consider (3). (The special case $i=1$ is also a special case of [3, Cor. 2, p. 23], because $M$ is a second syzygy module of itself over $A$.) By (2), $n$ is even. Hence, by $[5$, Cor.2.6, p. 462$]$,

$$
\sqrt{\operatorname{Fitt}_{2 i}^{R}(M)}=\sqrt{\operatorname{Fitt}_{2 i+1}^{R}(M)}
$$

and these two radicals are also equal to the radical of the ideal of $n-2 i$ Pfaffians of $\varphi$. The latter ideal has height at most $\left(\begin{array}{c}2 i+2 \\ 2\end{array}\right)$ by [20, Thm. 2.1, p. 191], where this statement is shown to follow from [8, pp. 202-3]. Since these radicals contain $\Delta$, we conclude that

$$
\text { height } \operatorname{Fitt}_{2 i}^{A}(M)=\text { height } \operatorname{Fitt}_{2 i+1}^{A}(M) \leq\left(\begin{array}{c}
2 i+2 \\
2
\end{array}\right)-1=2 i^{2}+3 i .
$$

Lemma 2.8. Let $R$ be a local ring, $B$ an $R$-algebra. Assume that, with respect to a set of generators $1, u_{2}, \ldots, u_{n}$, the $R$-module $B$ can be presented by a symmetric or alternating $n$ by $n$ matrix $\varphi$ with regular determinant. Set $F_{i}:=\operatorname{Fitt}_{i}^{R}(B)$. Let $\phi^{\prime}$ be the matrix obtained by deleting the first row and column of $\varphi$, and set $F_{i}^{\prime}:=\mathbf{I}_{n-i}\left(\phi^{\prime}\right)$. Then, for $i \geq 2$,

$$
\sqrt{F_{i}}=\sqrt{F_{i}^{\prime}}
$$

Proof. Let $p$ be a prime ideal of $R$ containing $F_{i}^{\prime}$. We need to show that $p$ contains $F_{i}$. Localizing at $p$, we may assume that $p$ is equal to the maximal ideal $m$ of $R$. If some entry of $\phi^{\prime}$ lies outside of $m$, then we may perform row and column operations to reduce the size of $\varphi$, until $\phi^{\prime}$ has all its entries in $m$. Furthermore, $F_{i}^{\prime} \neq R$; so $n-i \geq 1$. Hence $n-1 \geq i \geq 2$. Therefore, $F_{0} \subset m$, or equivalently, $B \neq 0$. 
Say $\varphi=\left(a_{i j}\right)$. We are going to show that the $a_{i j}$ lie in $m$. Suppose that $a_{1 j} \notin m$ for some $j$ with $2 \leq j \leq n$. Then $1 \in m B$ because the columns of $\varphi$ yield relations among $1, u_{2}, \ldots, u_{n}$ and because $\phi^{\prime}$ has all its entries in $m$. Hence $B=m B$. However, $B \neq 0$. Therefore, $a_{1 j} \in m$ for $2 \leq j \leq n$, and so $a_{j 1} \in m$ too because $\varphi$ is symmetric or alternating. Finally, repeating the argument with the first column of $\varphi$, we see that $a_{11} \in m$ as well. Thus $a_{i j} \in m$ for all $i, j$. So $F_{i} \subset m$ since $n-i \geq 1$.

Proposition 2.9. (1) Let $R$ be a Noetherian local ring, $B$ a finite $R$-algebra. Assume that $B$ is a perfect $R$-module of grade 1 and that $B$ is Gorenstein over $R$. Set $F_{i}:=\operatorname{Fitt}_{i}^{R}(B)$. Then, for $i \geq 2$, either height $F_{i} \leq\left(\begin{array}{c}i+1 \\ 2\end{array}\right)$ or $F_{i}=R$.

(2) Let $R$ be a regular local ring, $\Delta$ an $R$-regular element, and set $A:=R /(\Delta)$. Let $B$ be an $A$-algebra. Assume that $B$ is a finitely generated $A$-module of rank 2 and that $B$ is a Cohen-Macaulay ring. Set $F_{2}:=\operatorname{Fitt}_{2}^{R}(B)$. Then either height $F_{2} \leq 4$ or $F_{2}=R$.

Proof. In (1), the assertion follows from Theorem 2.3, from Lemma 2.8, and from [19, Thm. 2.1, p. 597] applied to the $n-1$ by $n-1$ matrix $\phi^{\prime}$ of Lemma 2.8.

Consider (2). Suppose that height $F_{2}>4$; then height $\operatorname{Fitt}_{2}^{A}(B)>3$. Hence, as an $A$-module, $B$ is free of rank 2 locally in codimension 3 . Therefore, the reflexive $A$-ideal $\left(\wedge^{2} B\right)^{* *}$ is principal locally in codimension 3 . Hence, $\left(\wedge^{2} B\right)^{* *}$ is globally principal by [16, Thm. 3.13(ii), p. 132]; in other words, $B$ is an orientable $A$-module. Therefore, Proposition 2.7 applies, and the assertion follows from Lemma 2.8 and [20, Thm. 2.1, p. 191].

Example 2.10. The bound in Proposition 2.9(1) can fail if $B$ isn't assumed to be Gorenstein over $R$, even for $i=2$. For example, let $n \geq 3$, let $R$ be the power series ring in $n(n-1)$ variables over a field, and let $I$ be the ideal of maximal minors of an $n$ by $n-1$ matrix $\phi$ in those variables. Let $\Delta \in I^{2}$, and let $B$ be the reciprocal of $I /(\Delta)$, viewed as a fractional ideal in the total ring of quotients of $R /(\Delta)$. Then $B$ is an $R$-algebra, which is a perfect $R$-module of grade 1 , by Parts (1) and (2) of Proposition 3.1 below. Moreover, the proof of Part (1) shows that $F_{2}$ may be described as the ideal of $n-2$ by $n-2$ minors of the $n$ by $n$ matrix obtained by adding to $\phi$ the column of combining coefficients in an expansion of $\Delta$ as a linear combination of the maximal minors of $\phi$. So $F_{2}$ contains the ideal of $n-2$ by $n-2$ minors of $\phi$. Therefore, height $F_{2} \geq 6$. This example also shows that Proposition 2.9(2) can fail if $B$ is of rank 1.

Lemma 2.11. Let $R$ be a Noetherian local ring. Let $B$ be an $R$-algebra, and assume that, as an $R$-module, $B$ is finitely generated and perfect of grade 1 . Set $A:=$ $R / \operatorname{Fitt}_{0}^{R}(B)$, and let $K$ and $L$ be the total rings of quotients of $A$ and $B$. Then the following six conditions are equivalent:
(i) $A \subset B$.
(iii) $K \rightarrow K \otimes_{A} B$.
(v) $B \subset K$.
(ii) $\operatorname{grade}\left(\operatorname{Fitt}_{1}^{R}(B)\right) \geq 2$.
(iv) $K=K \otimes_{A} B$.
(vi) $K=L$.

If $R$ satisfies Serre's condition $\left(\mathrm{S}_{2}\right)$, then the above six conditions are equivalent to the following one:

(vii) $\quad f: \operatorname{Spec}(B) \rightarrow \operatorname{Spec}(A)$ is birational onto its image.

Proof. Let $\varphi$ be an $n$ by $n$ matrix with entries in $R$ presenting $B$, and set $\Delta:=\operatorname{det} \varphi$. Then $\Delta$ is $R$-regular, and $(\Delta)=\operatorname{Fitt}_{0}^{R}(B)$. 
Conditions (i) and (ii) are equivalent. Indeed, (i) says that $(\Delta)=\operatorname{Ann}_{R}(B)$. However,

$$
\operatorname{Ann}(B)=(\Delta): \operatorname{Fitt}_{1}^{R}(B)
$$

by [4, Theorem, p. 232], which applies because $\Delta$ is an $R$-regular element contained in $\operatorname{Fitt}_{1}^{R}(B)$. Hence, (i) holds if and only if $\operatorname{Fitt}_{1}^{R}(B)$ is not contained in any associated prime of $(\Delta)$, and the latter condition is equivalent to (ii).

Conditions (ii) and (iii) are equivalent. Indeed, (ii) says that, for each $p \in$ $\operatorname{Ass}(A)$

$$
\operatorname{Fitt}_{1}^{A}(B)=\operatorname{Fitt}_{1}^{R}(B) \cdot A \not \subset p .
$$

Equivalently, the $A_{p}$-module $A_{p} \otimes_{A} B$ is cyclic. Or, equivalently again, the natural map from $A_{p}$ to $A_{p} \otimes_{A} B$ is surjective. The latter condition is simply (iii).

Trivially, (iv) implies (iii), and the converse holds because (iii) implies (i) and $K$ is $A$-flat.

Condition (iv) implies (v) because, in any event, $B \subset K \otimes_{A} B$. Indeed, take $p \in \operatorname{Ass}_{A}(B)$ and let $q$ be the preimage of $p$ in $R$. Since $B$ is a perfect $R$-module of grade one, depth $R_{q}=1$ by the Auslander-Buchsbaum formula. On the other hand, $p$ contains the $R$-regular element $\Delta$. Hence,

$$
q \in \operatorname{Ass}_{R}(R /(\Delta))=\operatorname{Ass}_{R}(A) .
$$

Therefore, $p \in \operatorname{Ass}_{A}(A)$. Consequently, every element of $A$ regular on $A$ is also regular on $B$, and so $B \subset K \otimes_{A} B$.

Condition (v) implies (i) because $A \subset K$. Condition (v) implies (vi). Indeed, if $B \subset K$, then every nonzerodivisor on $A$ is a nonzerodivisor on $B$, and so $B \subset K \subset$ $L$, whence $K=L$ since $K$ is its own total ring of quotients.

The map $f$ is locally of flat dimension 1 by the equivalence of (iii) and (vi) of $[22,(2.3)]$. Hence $f$ is locally of codimension 1 by $(2.5)(1)$ of $[22,(2.5)]$. By $[22,(3.2)(1)]$, therefore, $f$ is birational onto its image if and only if $\operatorname{Fitt}_{1}^{R}(B)$ is not contained in any minimal prime of $(\Delta)$. If $R$ satisfies $\left(\mathrm{S}_{2}\right)$, then $(\Delta)$ has only minimal associated primes. However, $\operatorname{Fitt}_{1}^{R}(B)$ is not contained in any associated prime of $(\Delta)$ if and only if Condition (ii) holds.

Proposition 2.12. Let $R$ be a Noetherian local ring, and $B$ an $R$-algebra. Assume that, as an $R$-module, $B$ is finitely generated and perfect of grade 1 . Assume that one of the equivalent conditions (i)-(vi) of Lemma 2.11 holds. Let $\varphi$ be an $n$ by $n$ matrix with entries in $R$ presenting $B$. Then there are invertible $n$ by $n$ matrices $\varepsilon$ and $\mu$ with entries in $R$ such that $\varepsilon \varphi \mu$ is symmetric if and only if $B$ is Gorenstein over $R$.

Proof. If there is a symmetric matrix $\varphi$ such that the sequence,

$$
0 \longrightarrow R^{n} \stackrel{\varphi}{\longrightarrow} R^{n} \longrightarrow B \longrightarrow 0,
$$

is exact, then there is an isomorphism of $A$-modules,

$$
\operatorname{Ext}_{R}^{1}(B, R) \cong B,
$$

and it is even $B$-linear since $A \subset B \subset K$, where $A$ and $K$ are as in Lemma 2.11. The converse holds by Theorem 2.3 . 


\section{SYMBOLIC POWERS}

In this section, we establish a bijective correspondence between the set of perfect algebras $B$ of grade 1 and the set of perfect ideals $I$ of grade 2 with a preferred member $\Delta$ of its second symbolic power, determined up to unit multiple; the first (resp., the second) Fitting ideal of $B$ must have grade at least 2 (resp., at least 3 ), and $I$ must be a complete intersection locally at each associated prime. The Gorenstein $B$ correspond to the self-linked $I$ with $\Delta$ as one of the self-linking elements. Proposition 3.1 starts with an $I$ and $\Delta$, takes $B$ to be the reciprocal of $I /(\Delta)$ in the total ring of quotients $K$ of $A:=R /(\Delta)$, and shows that $B$ has the requisite properties. Conversely, Proposition 3.6 starts with a $B$, takes $I$ to be its first Fitting ideal, and shows that $I$ has most of the requisite properties; the remaining ones follow from Proposition 3.1. We summarize the correspondence formally in Theorem 3.7. In addition, we investigate the symbolic Rees algebra $\mathcal{R}_{s} I$; notably, Theorem 3.4 gives a criterion in terms of $\mathcal{R}_{s} I$ for $I$ to be self-linked with $\Delta$ as one of the self-linking elements.

To recall the precise definitions, let $I$ be an ideal in a Noetherian ring $R$, set $S:=R / I$, let $W$ be the complement in $R$ of the union of all associated primes of $I$, and let $n \geq 0$ be an integer. Then the $n$th symbolic power $I^{(n)}$ of $I$ is the preimage of $I^{n} R_{W}$ in $R$. For $n>0$, notice that $I^{n} \subset I^{(n)} \subset I$ and that

$$
\left(I^{(n)} \cap I^{n-1}\right) / I^{n}
$$

is the $S$-torsion of $I^{n-1} / I^{n}$. The symbolic Rees algebra of $I$ is the graded subalgebra,

$$
\mathcal{R}_{s} I:=\bigoplus_{n \geq 0} I^{(n)} t^{n}
$$

of the polynomial ring $R[t]$ in one variable $t$. This algebra need not be Noetherian, even if $I$ happens to be a prime ideal in a regular ring; see [28], [29], and [14].

Proposition 3.1. Let $R$ be a Noetherian local ring, I a perfect ideal of grade 2, and $\Delta \in I$ a nonzerodivisor. Set $A:=R /(\Delta)$, and let " ${ }^{-}$indicate the image in $A$. Let $K$ be the total ring of quotients of $A$, and set $B:=A:_{K} \bar{I}$ and $F_{i}:=\operatorname{Fitt}_{i}^{R}(B)$.

(1) Then $\bar{I}$ and $B$ are perfect $R$-modules of grade 1 . In addition,

$$
\operatorname{Fitt}_{1}^{R}(I)=I, F_{0}=(\Delta), F_{i}=\operatorname{Fitt}_{i}^{R}(\bar{I}) \text {, and } \bar{I}=A:_{K} B .
$$

(2) Then the following four conditions are equivalent: (a) $B$ is a ring; (b) $B=$ $\bar{I}:_{K} \bar{I}$; (c) $B \bar{I}=\bar{I}$; and (d) $F_{1}=I$. Moreover, those four conditions are implied by this fifth condition: (e) $\Delta \in I^{(2)}$.

(2') If grade $F_{2} \geq 3$, then all five conditions (a) to (e) of (2) are equivalent.

(3) If $I$ is a complete intersection at each associated prime, then grade $F_{2} \geq 3$, and the converse holds if one of the conditions (a) to (e) of (2) holds.

(4) Assume that $B$ is a ring. Let $\alpha \in I$. Then the following conditions are equivalent: (i) $B$ is Gorenstein over $R$, and $\bar{\alpha} \notin \bar{I}$ n for any maximal ideal $n$ of $B$; (ii) $\bar{\alpha}$ is a nonzerodivisor and $B=\bar{\alpha}^{-1} \bar{I}$; (iii) $\bar{I}=\bar{\alpha} B$; (iv) $\bar{I}^{2}=\bar{\alpha} \bar{I}$; (v) $\bar{I}=(\bar{\alpha}):_{A} \bar{I}$; and (vi) $I=(\Delta, \alpha): I$.

Proof. Let $\phi$ be an $n$ by $n-1$ matrix with entries in $R$ presenting $I$. Let $\Delta_{1}, \ldots$, $\Delta_{n}$ be the signed maximal minors of $\phi$. They generate $I$ by the Hilbert-Burch Theorem; in other words, $\operatorname{Fitt}_{1}^{R}(I)=I$. So, since $\Delta$ is in $I$, there are elements $a_{1}$, 
$\ldots, a_{n}$ of $R$ such that $\Delta=\sum a_{i} \Delta_{i}$. Set

$$
\varphi:=\left(\begin{array}{cc}
a_{1} & \\
\vdots & \\
a_{n} &
\end{array}\right) .
$$

Then $\operatorname{det} \varphi=\Delta$. Furthermore, $\varphi$ presents $\bar{I}$. Hence, Lemma 2.5(1) yields

$$
\bar{I}=\operatorname{Ker}(\bar{\varphi}) \text { and } \operatorname{Hom}_{A}(\bar{I}, A)=\operatorname{Cok}\left(\bar{\varphi}^{*}\right) .
$$

Dualize the second equation and combine the result with the first; thus

$$
\operatorname{Hom}_{A}\left(\operatorname{Hom}_{A}(\bar{I}, A), A\right)=\bar{I} \text {. }
$$

On the other hand, $\operatorname{Cok}\left(\bar{\varphi}^{*}\right)=\operatorname{Cok}\left(\varphi^{*}\right)$ because $\Delta$ annihilates $\operatorname{Cok}\left(\varphi^{*}\right)$ since $\Delta=$ $\operatorname{det} \varphi^{*}$. Hence the second equation above implies that $\operatorname{Hom}_{A}(\bar{I}, A)$ is presented by $\varphi^{*}$ and is perfect of grade 1 . Finally, $\bar{I}$ contains a nonzerodivisor because grade $I \geq 2$; hence, there is a natural identification of $\operatorname{Hom}_{A}(\bar{I}, A)$ with $B$, and of $\operatorname{Hom}_{A}(B, A)$ with $A:_{K} B$; see the end of the proof of 2.5. Therefore, (1) holds.

Consider (2). Obviously, (c) implies (b), and (b) implies (a); also, (a) implies (c) because $\bar{I}=A:_{K} B$ by (1). Now, it follows from the preceding paragraph that

$$
F_{1}=\mathbf{I}_{n-1}(\varphi), I=\mathbf{I}_{n-1}(\phi), \operatorname{Hom}_{A}(\bar{I}, A)=B \text { and } \operatorname{Hom}_{A}(\bar{I}, \bar{I})=\bar{I}:_{K} \bar{I} .
$$

Hence, (d) and (b) are equivalent by Lemma 2.5(2). Thus the four conditions (a) to (d) are equivalent.

Finally, assume (e), that $\Delta \in I^{(2)}$. We'll prove (d), that $F_{1}=I$. Now,

$$
F_{1}=\mathbf{I}_{n-1}\left(\varphi^{*}\right) \supset \mathbf{I}_{n-1}(\phi)=I .
$$

Hence, it suffices to prove (d) after localizing at an arbitrary associated prime of $I$. But then $I^{(2)}=I^{2}$, so $\Delta \in I^{2}$. Hence we may assume that $a_{i} \in I$ because the $\Delta_{i}$ generate $I$. Therefore,

$$
F_{1}=\mathbf{I}_{n-1}\left(\varphi^{*}\right) \subset \mathbf{I}_{n-1}(\phi)+I=I .
$$

Hence $F_{1}=I$. Thus (2) holds.

Consider $\left(2^{\prime}\right)$. The Laplace identity (2.4) implies that $F_{2}(\Delta) \subset F_{1}^{2}$. Hence, if $F_{1}=I$, then $\Delta \in I^{(2)}$ because $F_{2}$ lies in no associated prime of $I$ as $I$ is perfect of grade 2 and grade $F_{2} \geq 3$.

Consider (3). First assume that $I$ is a complete intersection at each associated prime. Since $I$ is perfect of grade 2 , every prime $p$ such that $p \supset I$ and depth $R_{p}=$ 2 is associated to $I$. Hence $I_{p}$ can be generated by two elements. Therefore, $\operatorname{grade} \operatorname{Fitt}_{2}^{R}(I) \geq 3$. Hence, grade $F_{2} \geq 3$ because

$$
F_{2}=\mathbf{I}_{n-2}\left(\varphi^{*}\right) \supset \mathbf{I}_{n-2}(\phi)=\operatorname{Fitt}_{2}^{R}(I) .
$$

Conversely, assume that grade $F_{2} \geq 3$ and that one of the conditions (a) to (e) of (2) holds. Then $\Delta \in I^{(2)}$ by $\left(2^{\prime}\right)$. Now, let $p \in \operatorname{Ass}(I)$, and replace $R$ by $R_{p}$. Then $I^{(2)}=I^{2}$. In addition, $F_{2}=R$ because grade $F_{2} \geq 3$, but depth $R_{p}=2$. Hence (1) implies that $\bar{I}$ is generated by two elements. Lift them to $I$. Those lifts generate $I$ because $\Delta \in I^{(2)}=I^{2}$. Hence the lifts form a regular sequence because grade $I=2$.

Consider (4). The canonical isomorphisms,

$$
\operatorname{Ext}_{R}^{1}(B, R)=\operatorname{Hom}_{A}(B, A)=A:_{K} B=\bar{I},
$$


are obviously $B$-linear. Hence, $B$ is Gorenstein over $R$ if and only if $\bar{I}$ is $B$ isomorphic to $B$. Therefore, (i) implies (iii), and the converse holds because $\bar{I}$ contains a nonzerodivisor. Obviously, (ii) and (iii) are equivalent. Obviously, (iii) implies (iv). Suppose (iv) holds. Then $\bar{\alpha}$ is a nonzerodivisor, because $\bar{\alpha}$ divides the square of any element of $\bar{I}$, and $\bar{I}$ contains a nonzerodivisor. So $(\bar{\alpha}-1 \bar{I}) \bar{I}=\bar{I}$. So $\bar{\alpha}^{-1} \bar{I} \subset B$ because $B=\bar{I}:_{K} \bar{I}$ by (2). However, $\bar{\alpha} B \subset \bar{I}$ because $\alpha \in I$; so $B \subset \bar{\alpha}^{-1} \bar{I}$. So (ii) holds. Thus (i) to (iv) are equivalent.

If (v) holds, then $\bar{\alpha}$ is a nonzerodivisor, because, again, $\bar{\alpha}$ divides the square of any element of $\bar{I}$. Now, whenever $\bar{\alpha}$ is a nonzerodivisor, then, clearly,

$$
(\bar{\alpha}):_{A} \bar{I}=(\bar{\alpha}):_{K} \bar{I}=\bar{\alpha}\left(A:_{K} \bar{I}\right)=\bar{\alpha} B \text {. }
$$

Hence, (iii) and (v) are equivalent. Obviously, (v) and (vi) are equivalent.

Lemma 3.2. Let $R$ be a Noetherian local ring with infinite residue field, and let $I$ be an ideal of grade 2 that is a complete intersection at each associated prime. Assume that the analytic spread $\ell\left(I^{(2)}\right)$ of $I^{(2)}$ is equal to 2 , and that $I^{(2)} / I^{2}$ is a cyclic module. Let $\Delta \in I^{(2)}$ be a general element, and $\alpha \in I$ an element whose image in $I /(\Delta)$ is general. Then $I=(\Delta, \alpha): I$.

Proof. Let " ${ }^{-}$' indicate the image in $R /(\Delta)$. Now, since $\Delta \in I^{(2)}$ is general, it is part of a set of $\ell\left(I^{(2)}\right)$ elements generating a reduction $J$ of $I^{(2)}$. Then $\bar{J}$ is a reduction of $\overline{I^{(2)}}$, and so $\bar{J}$ requires at least $\ell\left(\overline{I^{(2)}}\right)$ generators. Hence $\ell\left(\overline{I^{(2)}}\right) \leq \ell\left(I^{(2)}\right)-1$, and therefore $\ell\left(\overline{I^{(2)}}\right)=1$. On the other hand, $I^{(2)}=I^{2}+(\Delta)$; hence, $\overline{I^{(2)}}=\overline{I^{2}}$. Therefore, $\ell\left(\bar{I}^{2}\right)=1$. Consequently, $\ell(\bar{I})=1$. Since $\bar{\alpha} \in \bar{I}$ is general, therefore $\bar{\alpha}$ generates a reduction of $\bar{I}$. In particular, $\bar{I}$ and $\bar{\alpha}$ have the same radical. So $I$ and $(\Delta, \alpha)$ have the same radical too. Therefore, every associated prime $p$ of $(\Delta, \alpha)$ is one of $I$; indeed, depth $R_{p}=2$ and $p \supset I$, whence $\operatorname{depth}(R / I)_{p}=0$ since $I$ is perfect of grade 2 .

It suffices to check the equation $I=(\Delta, \alpha): I$ locally at each of the (finitely many) associated primes $p$ of $I$, since any associated prime of $(\Delta, \alpha): I$ is one of $(\Delta, \alpha)$, so one of $I$. Localizing at $p$, we may assume that $I$ is a complete intersection. Let $m$ denote the maximal ideal of $R$. Then $\bar{\alpha} \notin \bar{m} \bar{I}$ because $\bar{\alpha} \in \bar{I}$ is general. So $\alpha \notin m I$. Hence $I=(\alpha, \beta)$ for some $\beta$, and $\alpha, \beta$ form a regular sequence. Hence the associated graded $\operatorname{ring} \operatorname{gr}_{I}(R)$ is a polynomial ring over $R / I$; see [25, p. 125] for instance. In particular, $\operatorname{gr}_{I}(R)$ is torsion free over $R / I$; hence, $I^{(2)}=I^{2}$. Therefore, $\Delta \in I^{2}$. So $\Delta=r \alpha^{2}+s \alpha \beta+t \beta^{2}$ for some $r, s, t$. Now, $\sqrt{I}=\sqrt{(\Delta, \alpha)}$, so $I^{i}=(\Delta, \alpha) \cap I^{i}$ for some $i$. On the other hand, by the generality of $\Delta$ and $\alpha$, their leading forms in $\operatorname{gr}_{I}(R)$ have degrees 2 and 1, and they form a regular sequence; it follows that $(\Delta, \alpha) \cap I^{i}=\Delta I^{i-2}+\alpha I^{i-1}$ (see [31, Thm. 2.3, p. 97] for instance). Hence $I^{i}=\Delta I^{i-2}+\alpha I^{i-1}$. Suppose $t \in m$. Then $I^{i}=\alpha I^{i-1}+t I^{i}$. So Nakayama's lemma implies that $I^{i}=\alpha I^{i-1}$. Hence $I^{i} \subset(\alpha)$. However, this inclusion is impossible because $I^{i}$ has grade 2 . Thus $t \notin m$. Hence $(\Delta, \alpha)=\left(\alpha, \beta^{2}\right)$. Hence,

$$
(\Delta, \alpha): I=\left(\alpha, \beta^{2}\right):(\alpha, \beta)=(\alpha, \beta)=I \text {. }
$$

Proposition 3.3. Let $R$ be a Noetherian local ring, I a perfect ideal of grade 2 . Assume that $I$ is a complete intersection at each associated prime, and that $I=$ $(\Delta, \alpha): I$ for some $\Delta \in I^{(2)}$ and some $\alpha \in I$. 
(1) If also $R$ has an infinite residue field, then $I=(\Delta, \alpha): I$ for any general $\Delta \in I^{(2)}$ and any $\alpha \in I$ whose image in $I /(\Delta)$ is general.

(2) Then $I^{(2)} / I^{2}$ is cyclic, and $\ell\left(I^{(i)}\right)=2$ for every even $i>0$.

(3) Then $I^{(i)}$ is a self-linked perfect ideal for every $i>0$.

(4) Then the symbolic Rees algebra $\mathcal{R}_{s} I$ is Noetherian; in fact,

$$
\mathcal{R}_{s} I=R\left[I t, \Delta t^{2}\right] .
$$

Proof. Set $A:=R /(\Delta)$ and let " - indicate the image in $A$. First, we show that, for every $i \geq 2$,

$$
I^{(i)}=\alpha^{i-1} I+\left((\Delta) \cap I^{(i)}\right) .
$$

That equation is equivalent to this one: $\overline{I^{(i)}}=\bar{\alpha}^{i-1} \bar{I}$. The latter may be checked at each associated prime $p$ of the $R$-module $\overline{I^{(i)}} / \bar{\alpha}^{i-1} \bar{I}$, which is a submodule of $A / \bar{\alpha}^{i-1} \bar{I}$. So $p$ must be an associated prime of $A / \bar{\alpha}^{i-1} \bar{I}$. So $\left(A / \bar{\alpha}^{i-1} \bar{I}\right)_{p}$ has depth 0 . Hence $\left(\bar{\alpha}^{i-1} \bar{I}\right)_{p}$ has depth 1 . Hence $\bar{I}_{p}$ has depth 1 . Now, $A / \bar{I}=R / I$, and so there is a short exact sequence,

$$
0 \rightarrow \bar{I}_{p} \rightarrow A_{p} \rightarrow(R / I)_{p} \rightarrow 0 .
$$

Therefore, either depth $A_{p} \leq 1$ or $\operatorname{depth}(R / I)_{p}=0$. In the former case, depth $R_{p} \leq$ 2. Hence, again $\operatorname{depth}(R / I)_{p}=0$. So, in any case, either $I \not \subset p$, or $p$ is an associated prime of the ideal $I$. Hence $\left(I^{(i)}\right)_{p}=\left(I^{i}\right)_{p}$. On the other hand, Parts (2) and (4) of Proposition 3.1 yield $\bar{I}^{i}=\bar{\alpha}^{i-1} \bar{I}$. Hence $\left(\overline{I^{(i)}}\right)_{p}=\left(\bar{\alpha}^{i-1} \bar{I}\right)_{p}$, and the proof of (3.3.1) is complete.

Next, we show that, for every $i \geq 2$,

$$
(\Delta) \cap I^{(i)}=\Delta I^{(i-2)} .
$$

First, consider the case where $I$ is generated by a regular sequence. Suppose that $\Delta \in m I^{2}$, where $m$ denotes the maximal ideal of $R$. Then

$$
I^{2}=I^{2} \cap(\Delta, \alpha)=I^{2} \cap\left(m I^{2}, \alpha\right)=m I^{2}+(\alpha) \cap I^{2} .
$$

So Nakayama's lemma implies that $I^{2}=(\alpha) \cap I^{2} \subset(\alpha)$. However, this inclusion is impossible because $I^{2}$ has height 2. Thus $\Delta \in I^{2} \backslash m I^{2}$. Hence, in the associated graded ring $\operatorname{gr}_{I} R$, the leading form of $\Delta$ is a regular element, because $\operatorname{gr}_{I} R$ is a polynomial ring. Consequently, $(\Delta) \cap I^{i}=\Delta I^{i-2}$. Thus (3.3.2) holds for complete intersections.

To prove (3.3.2) in general, it suffices to show that $(\Delta) \cap I^{(i)}$ lies in $\Delta I^{(i-2)}$. So let $p$ be an associated prime of the ideal $\Delta I^{(i-2)}$. We may assume that $I \subset p$, since the assertion is trivial otherwise. But, then

$$
\operatorname{depth}\left(I^{(i-2)}\right)_{p}=\operatorname{depth}\left(\Delta I^{(i-2)}\right)_{p}=1 .
$$

Hence $p$ is an associated prime of the ideal $I^{(i-2)}$ (and so $i-2 \geq 1$ ). Therefore, by the definition of symbolic powers, $p$ is contained in the union of the associated primes of the ideal $I$; so $p$ is contained in one of them. Hence, again, $I_{p}$ is generated by a regular sequence, and so the assertion follows from the discussion in the preceding paragraph.

Together, (3.3.1) and (3.3.2) yield the following equation: for $i \geq 2$,

$$
I^{(i)}=\alpha^{i-1} I+\Delta I^{(i-2)} .
$$


In turn, that equation yields, via induction on $j$, this one: for $j \geq 1$,

$$
I^{(2 j)}=\alpha\left(\Delta, \alpha^{2}\right)^{j-1} I+\left(\Delta^{j}\right) .
$$

On the other hand, (3.3.3) immediately yields Assertion (4).

To prove Assertions (1) and (2), note that $I^{(2)}=\alpha I+(\Delta)$ by (3.3.3). So $I^{(2)} / I^{2}$ is cyclic. Furthermore,

$$
\left(I^{(2)}\right)^{2}=\alpha^{2} I^{2}+\Delta \alpha I+\left(\Delta^{2}\right) \subset\left(\Delta, \alpha^{2}\right) I^{(2)} \subset\left(I^{(2)}\right)^{2} .
$$

Hence $\left(I^{(2)}\right)^{2}=\left(\Delta, \alpha^{2}\right) I^{(2)}$. Since $\left(\Delta, \alpha^{2}\right)$ lies in $I^{(2)}$, it is therefore a reduction of $I^{(2)}$. Hence $\ell\left(I^{(2)}\right)=2$. Therefore, Lemma 3.2 implies Assertion (1). Moreover, similarly, (3.3.4) implies that $\left(\Delta^{j}, \alpha^{2 j}\right)$ is a reduction of $I^{(2 j)}$; hence, $\ell\left(I^{(2 j)}\right)=2$. Thus Assertion (2) holds.

Together, (3.3.2) and (3.3.3) yield the following short exact sequence for $i \geq 2$ :

$$
0 \rightarrow I^{(i-2)} \stackrel{\Delta}{\rightarrow} I^{(i)} \rightarrow \bar{\alpha}^{i-1} \bar{I} \rightarrow 0
$$

Since the $R$-module $I$ has projective dimension at most 1 , so do $\bar{I}$ and therefore also $\bar{\alpha}^{i-1} \bar{I}$. Hence it follows from (3.3.5), via induction on $i$, that $I^{(i)}$ too has projective dimension at most 1 . Therefore, $I^{(i)}$ is perfect, as asserted in (3).

To prove that $I^{(i)}$ is self-linked, we first treat the case where $i$ is odd, say, $i=2 j-1$ with $j \geq 1$. In fact, we claim that

$$
I^{(2 j-1)}=\left(\Delta^{j}, \alpha^{2 j-1}\right): I^{(2 j-1)} .
$$

Now, $I=(\Delta, \alpha): I$. So $I^{2} \subset(\Delta, \alpha) \subset I$. Hence $I$ and $(\Delta, \alpha)$ have the same radical. Hence $I^{2 j-1}$ and $\left(\Delta^{j}, \alpha^{2 j-1}\right)$ have the same radical as $I$. Therefore, it suffices to establish (3.3.6) locally at each associated prime $p$ of $I$. However, by localizing at $p$, we may assume that $I^{(2 j-1)}=I^{2 j-1}$, that $I=(\alpha, \beta)$, and that $\Delta=r \alpha^{2}+s \alpha \beta+t \beta^{2}$ for some $r, s, t$. Here $t$ has to be a unit; otherwise, $I^{(2)} \neq \alpha I+(\Delta)$, contradicting (3.3.3). So we may suppose that $\Delta=r \alpha^{2}+s \alpha \beta+\beta^{2}$.

We thus need to show that

$$
\left(\Delta^{j}, \alpha^{2 j-1}\right):(\alpha, \beta)^{2 j-1}=(\alpha, \beta)^{2 j-1},
$$

where $\Delta^{j}=\sum_{k=0}^{2 j-2} b_{k} \alpha^{2 j-1-k} \beta^{k}+\beta \beta^{2 j-1}$ with $b_{k} \in(\alpha, \beta)$. Now, $(\alpha, \beta)^{2 j-1}$ is generated by the signed maximal minors $d_{1}, \ldots, d_{2 j}$ of the following $2 j$ by $2 j-1$ matrix:

$$
\left(\begin{array}{cccc}
-\beta & & & \\
\alpha & -\beta & & \\
& \alpha & \ddots & \\
& & \ddots & -\beta \\
& & & \alpha
\end{array}\right) .
$$


Adjoin the two columns of coefficients that arise when $\Delta^{i}$ and $\alpha^{2 j-1}$ are written in terms of the minors $d_{1}, \ldots, d_{2 j}$, obtaining the following $2 j$ by $2 j+1$ matrix:

$$
\left(\begin{array}{cccccc}
-\beta & & & & b_{0} & 1 \\
\alpha & -\beta & & & b_{1} & 0 \\
& \alpha & \ddots & & \vdots & \vdots \\
& & \ddots & -\beta & b_{2 j-2} & 0 \\
& & & \alpha & \beta & 0
\end{array}\right) .
$$

Its maximal minors generate the link $\left(\Delta^{j}, \alpha^{2 j-1}\right):(\alpha, \beta)^{2 j-1}$; see $[1, \mathrm{p} .316]$. The preceding matrix may be reduced to the following one:

$$
\left(\begin{array}{ccccc}
\alpha & -\beta & & & b_{1} \\
& \alpha & \ddots & & \vdots \\
& & \ddots & -\beta & b_{2 j-2} \\
& & & \alpha & \beta
\end{array}\right) .
$$

Denote the minor obtained by deleting the $i$ th column by $D_{i}$. Then

$$
\left(D_{l}, \ldots, D_{2 j}\right)=\left(\alpha^{l-1} \beta^{2 j-l}, \ldots, \alpha^{2 j-1}\right)
$$

for $1 \leq l \leq 2 j$, as can be proved easily via descending induction on $l$ because $b_{k} \in(\alpha, \beta)$. In particular, $\left(D_{1}, \ldots, D_{2 j}\right)=(\alpha, \beta)^{2 j-1}$. Thus the asserted equation (3.3.7) holds.

If $i$ is even, say $i=2 j$ with $j \geq 1$, then we claim that

$$
I^{(2 j)}=\left(\Delta^{j}, \alpha^{2 j+1}\right): I^{(2 j)} .
$$

Set $C:=R /\left(\Delta^{j}\right)$; let ${ }^{~} \sim$ ' indicate the image in $C$; and let $L$ denote the total ring of quotients of $C$. In these terms, our claim asserts that $\widetilde{I^{(2 j)}}$ is equal to $\left(\widetilde{\alpha}^{2 j+1}\right):_{C} \widetilde{I^{(2 j)}}$. However,

$$
\begin{aligned}
\widetilde{I^{(2 j)}} & =\widetilde{\alpha} I^{(2 j-1)} \\
& =\widetilde{\alpha}\left(\left(\widetilde{\alpha}^{2 j-1}\right):_{C} \widetilde{I^{(2 j-1)}}\right) \\
& =\widetilde{\alpha}\left(\left(\widetilde{\alpha}^{2 j-1}\right):_{L} \widetilde{I^{(2 j-1)}}\right) \\
& =\left(\widetilde{\alpha}^{2 j+1}\right):_{L}\left(\widetilde{\alpha I^{(2 j-1)}}\right) \\
& =\left(\widetilde{\alpha}^{2 j+1}\right):_{C}\left(\widetilde{\alpha} \widetilde{I^{(2 j-1)}}\right) \\
& =\left(\widetilde{\alpha}^{2 j+1}\right):_{C} \widetilde{I^{(2 j)}} .
\end{aligned}
$$

Indeed, the first and the last equations hold by (3.3.4); the second equation holds by the case of an odd $i$; the third and fifth equations hold because $\widetilde{\alpha}$ lies in $\widetilde{I}$ and is a nonzerodivisor; and the fourth equation holds because $\widetilde{\alpha}$ is a nonzerodivisor. Thus the assertion about self-linkage holds. So Assertion (3) holds, and the proof of 3.3 is complete.

Theorem 3.4. Let $R$ be a Cohen-Macaulay local ring with infinite residue field, and I a perfect ideal of grade 2 that is a complete intersection at each associated prime. Then the following conditions are equivalent:

(i) $I=(\Delta, \alpha): I$ for some $\Delta \in I^{(2)}$ and some $\alpha \in I$; 
(i') $I=(\Delta, \alpha): I$ for any general $\Delta \in I^{(2)}$ and any $\alpha \in I$ whose image in $I /(\Delta)$ is general;

(ii) $I^{(2)} / I^{2}$ is cyclic, and $\ell\left(I^{(2)}\right)=2$;

(iii) $\mathcal{R}_{s} I=R\left[I t, \Delta t^{2}\right]$ for some $\Delta \in I^{(2)}$, and $I^{(i)}$ is a perfect ideal for every $i>0$

(iv) $\mathcal{R}_{s} I=R\left[I t, \Delta t^{2}\right]$ for some $\Delta \in I^{(2)}$, and $I^{(i)}$ is a Cohen-Macaulay ideal for infinitely many $i>0$.

Moreover, if one of the preceding conditions obtains, then $I^{(i)}$ is self-linked for every $i>0$, and $\mathcal{R}_{s} I$ is a Cohen-Macaulay ring.

Proof. First, (ii) implies (i') by Lemma 3.2, and (i') implies (i) trivially. Second, (i) implies (ii) and (iii), and (i) implies that $I^{(i)}$ is self-linked, all by Proposition 3.3. (Those implications do not require the Cohen-Macaulay hypothesis, but the remaining implications do.) Third, clearly, (iii) implies (iv). Fourth, assume (iv). Then $\mathcal{R}_{s} I=R\left[I t, I^{(2)} t^{2}\right]$; hence, $I^{(2 i)}=\left(I^{(2)}\right)^{i}$ for every $i>0$. Therefore, infinitely many powers of $I^{(2)}$ are Cohen-Macaulay ideals of height 2 . Hence, $\ell\left(I^{(2)}\right)=2$; see $\left[2\right.$, Thm. 2, p. 36]. On the other hand, $I^{(2)}=I^{2}+(\Delta)$; so $I^{(2)} / I^{2}$ is cyclic. Thus (ii) holds, and so (i) to (iv) are equivalent. Finally, (i) implies that $\mathcal{R}_{s} I$ is Cohen-Macaulay by the next proposition.

Proposition 3.5. Let $R$ be a Noetherian local ring, I a perfect ideal of grade 2. Assume that $I$ is a complete intersection at each associated prime, and that $I=(\Delta, \alpha): I$ for some $\Delta \in I^{(2)}$ and some $\alpha \in I$. If $R$ is Cohen-Macaulay or Gorenstein, then so is $\mathcal{R}_{s} I$.

Proof. Set $A:=R /(\Delta)$ and let " ', indicate the image in $A$. Form the graded ring $G:=\bigoplus_{i \geq 0} I^{(i)} / I^{(i+1)}$ associated to the filtration $\left\{I^{(i)} \mid i \geq 0\right\}$ of $R$. Denote the leading form of $\Delta$ in $G$ by $\Delta^{\prime}$. Then $\operatorname{deg} \Delta^{\prime} \geq 2$ since $\Delta \in I^{(2)}$. On the other hand, for every $i \geq 1$,

$$
\Delta\left(I^{(i+1)}:(\Delta)\right)=(\Delta) \cap I^{(i+1)}=\Delta I^{(i-1)} ;
$$

indeed, the first equation is obvious, and the second is (3.3.2). Hence

$$
I^{(i+1)}:(\Delta)=I^{(i-1)}
$$

because $\Delta$ is $R$-regular. Therefore, $\operatorname{deg} \Delta^{\prime}=2$ and $\Delta^{\prime}$ is $G$-regular. Furthermore, again by (3.3.2), for every $i \geq 2$,

$$
I^{(i+1)}+\Delta I^{(i-2)}=I^{(i+1)}+\left((\Delta) \cap I^{(i)}\right) .
$$

Therefore, $G /\left(\Delta^{\prime}\right)=\bigoplus_{i \geq 0} \overline{I^{(i)}} / \overline{I^{(i+1)}}$. Finally, (3.3.3) gives $\overline{I^{(i)}}=\overline{I^{i}}$ for every $i \geq 2$; in fact, then, obviously, this equation holds for every $i \geq 0$. Therefore,

$$
G /\left(\Delta^{\prime}\right)=\bigoplus_{i \geq 0} \overline{I^{(i)}} / \overline{I^{(i+1)}}=\bigoplus_{i \geq 0} \overline{I^{i}} / \overline{I^{i+1}}=\operatorname{gr}_{I} A .
$$

Thus there is a short exact sequence of graded $G$-modules,

$$
0 \rightarrow G(-2) \stackrel{\Delta^{\prime}}{\rightarrow} G \rightarrow \operatorname{gr}_{\bar{I}} A \rightarrow 0
$$

Let $\bar{\alpha}^{\prime}$ denote the leading form of $\bar{\alpha}$ in $\operatorname{gr}_{\bar{I}} A$. Then $\bar{\alpha}^{\prime}$ is a regular element on $\operatorname{gr}_{\bar{I}} A$ of degree one; indeed, $\overline{I^{i+1}}:(\bar{\alpha})=\overline{I^{i}}$, because $\bar{\alpha}$ is an $A$-regular element contained in $\bar{I}$ and because $\bar{I}^{i+1}=\bar{\alpha} \bar{I}^{i}$ for every $i \geq 1$ by (3.3.3). Moreover, $\bar{\alpha} \bar{I}^{i-1}$ is equal to $\bar{\alpha} \cap \bar{I}^{i}$ because the latter is equal to $\bar{\alpha}\left(\overline{I^{i}}:(\bar{\alpha})\right)$. Therefore, $\operatorname{gr}_{\bar{I}} A /\left(\bar{\alpha}^{\prime}\right)$ can 
be identified with the associated graded ring of the ideal $\widetilde{I}:=I /(\Delta, \alpha)$ in $R /(\Delta, \alpha)$. The latter graded ring is equal to the trivial extension $S \ltimes \widetilde{I}(-1)$ of $S:=R / I$ because $\widetilde{I}^{2}=0$. Thus there is an exact sequence of graded $G$-modules,

$$
0 \rightarrow \operatorname{gr}_{\bar{I}} A(-1) \stackrel{\bar{\alpha}^{\prime}}{\rightarrow} \operatorname{gr}_{\bar{I}} A \rightarrow S \ltimes \widetilde{I}(-1) \rightarrow 0 .
$$

Sequences (3.5.1) and (3.5.2) say, in other words, that the leading forms $\Delta^{\prime}$ and $\alpha^{\prime}$ of $\Delta$ and $\alpha$ in $G$ have degrees two and one, that they form a regular sequence, and that $G /\left(\Delta^{\prime}\right)$ is equal to $\operatorname{gr}_{\bar{I}} A$ and $G /\left(\Delta^{\prime}, \alpha^{\prime}\right)$ is equal to $S \ltimes \widetilde{I}(-1)$.

Set $d:=\operatorname{dim} R$. Let $m$ denote the maximal ideal of $R$, and $\mathbf{M}$ the irrelevant maximal ideal of $G$. Given a finitely generated graded $G$-module of dimension $s$, let $H_{\mathbf{M}}^{j}(E)$ denote the $j$ th local cohomology module of $E$ with supports in $\mathbf{M}$, and set

$$
a(E):=\sup \left\{i \mid\left[H_{\mathbf{M}}^{s}(E)\right]_{i} \neq 0\right\} .
$$

Assume now that $R$ is a Cohen-Macaulay ring. Then $S$ is a Cohen-Macaulay ring, and $\widetilde{I}$ a maximal Cohen-Macaulay $S$-module because $I$ is a perfect $R$-ideal of grade 2; hence, $S \ltimes \widetilde{I}(-1)$ is a Cohen-Macaulay ring. Therefore, $\operatorname{gr}_{\bar{I}} A$ and $G$ are Cohen-Macaulay too, because $\Delta^{\prime}$ and $\alpha^{\prime}$ form a regular sequence. Furthermore,

$$
\begin{aligned}
H_{\mathrm{M}}^{d-2}(S \ltimes \widetilde{I}(-1)) & =H_{m G}^{d-2}(S \ltimes \widetilde{I}(-1))=H_{m}^{d-2}(S \ltimes \widetilde{I}(-1)) \\
& =H_{m}^{d-2}(S) \oplus H_{m}^{d-2}(\widetilde{I})(-1) .
\end{aligned}
$$

Hence $a(S \ltimes \widetilde{I}(-1))=1$. Hence (3.5.2) implies $a\left(\operatorname{gr}_{\bar{I}} A\right)=0$ because $\operatorname{gr}_{\bar{I}} A$ is Cohen-Macaulay. Hence (3.5.1) implies $a(G)=-2$ because $G$ is Cohen-Macaulay. Now, by $\left[12,(1.2)\right.$, p. 74], since $a(G)<0$ and since $G$ is Cohen-Macaulay, $\mathcal{R}_{s} I$ is Cohen-Macaulay.

Suppose $R$ is Gorenstein. Then the dualizing module $\omega_{S}$ of $S$ is isomorphic to $\operatorname{Ext}_{R}^{2}(S, R)$. However, $\Delta, \alpha$ form a regular sequence, so this Ext is equal to $\operatorname{Hom}_{R}(S, R /(\Delta, \alpha))$ or, what is the same, to

$$
((\Delta, \alpha): I) /(\Delta, \alpha) \text {. }
$$

This module is equal to $\widetilde{I}$. Thus $\widetilde{I} \cong \omega_{S}$. So $S \ltimes \widetilde{I} \cong S \ltimes \omega_{S}$. The latter is a Gorenstein ring (see $[27,(7)$, p. 419] for instance). Hence $G$ is Gorenstein too, because $\Delta^{\prime}$ and $\alpha^{\prime}$ form a regular sequence. Finally, by [12, (1.4), p. 75], since $a(G)=-2$ and since $G$ is Gorenstein, $\mathcal{R}_{s} I$ is Gorenstein.

Proposition 3.6. Let $R$ be a Noetherian local ring, and $B$ an $R$-algebra. Assume that, as an R-module, $B$ is finitely generated and perfect of grade 1 . Set $F_{i}:=$ $\operatorname{Fitt}_{i}^{R}(B)$. Set $I:=F_{1}$, and assume $I \neq R$ and grade $I \geq 2$. Set $A:=R / F_{0}$ and let ${ }^{\prime-}$ ' indicate the image in $A$. Given generators $1, u_{2}, \ldots, u_{n}$ of the $R$-module $B$, let $\varphi$ be an $n$ by $n$ matrix with entries in $R$ whose transpose $\varphi^{*}$ presents $B$ via those generators. Set $\Delta:=\operatorname{det} \varphi$. Let $\phi$ be the $n$ by $n-1$ matrix consisting of the last $n-1$ columns of $\varphi$, and set $I^{\prime}:=\mathbf{I}_{n-1}(\phi)$.

(1) Then $I=I^{\prime}$; moreover, $I$ is a perfect $R$-ideal of grade 2 , and is presented by $\phi$. Furthermore, $\Delta$ is regular. In addition, if $K$ denotes the total ring of quotients of $A$, then

$$
A \subset B \subset K, \bar{I}=A:_{K} B \text {, and } B=A:_{K} \bar{I} \text {. }
$$

(2) Assume that grade $F_{2} \geq 3$. Then $I$ is a complete intersection at each associated prime, and $\Delta \in I^{(2)}$. 
(3) Assume that $B$ is Gorenstein over $R$, and modify $\varphi$ to make it symmetric (see Theorem 2.3). Let $\phi^{\prime}$ be the $n-1$ by $n-1$ matrix obtained by deleting the first row and column of $\varphi$, and set $\alpha:=\operatorname{det} \phi^{\prime}$ and $F_{2}^{\prime}:=\mathbf{I}_{n-2}\left(\phi^{\prime}\right)$. Then $\Delta, \alpha$ form an $R$-regular sequence, with respect to which $I$ is self-linked. Moreover, $\bar{I}^{i+1}=\bar{\alpha} \bar{I}^{i}$ for every $i \geq 1$, and $B=\bar{\alpha}^{-1} \bar{I}$. In addition,

$$
F_{2}=\operatorname{Fitt}_{2}^{R}(I)=I^{2}:(\Delta)=F_{2}^{\prime} .
$$

Proof. Consider (1). First of all, the proof of 2.8 yields, mutatis mutandis, that $\sqrt{I}=\sqrt{I^{\prime}}$. Hence grade $I^{\prime} \geq 2$. Therefore, by the Hilbert-Burch theorem, $I^{\prime}$ is a perfect ideal of grade 2 , and is presented by $\phi$. Second, $\varphi^{*}$ presents $B$, so $B=\operatorname{Cok}\left(\bar{\varphi}^{*}\right)$. Moreover, $B$ is perfect of grade 1 ; hence $\varphi^{*}$ is injective and so $\Delta$ is regular. Finally, consider the proof of 3.1(1). The latter half applies, therefore, to the present $\varphi$, and yields $B=A:_{K} \overline{I^{\prime}}$ and $\overline{I^{\prime}}=A:_{K} B$. Hence Proposition 3.1(2) yields $I=I^{\prime}$. Thus (1) is proved.

Assertion (2) follows from Parts $\left(2^{\prime}\right)$ and (3) of Proposition 3.1, which applies to the present $I$ and $\Delta$ because of $(1)$.

Consider (3). The first assertions follow, thanks to (1), from Lemma 2.5(3) and the equivalence of (vi), (iv), and (ii) of Proposition 3.1(4). Now, $\phi$ presents $I$ by (1); hence,

$$
F_{2}^{\prime} \subset \operatorname{Fitt}_{2}^{R}(I) \subset F_{2} .
$$

On the other hand, we're about to show that

$$
F_{2} \subset F_{1}^{2}:(\Delta) \subset F_{2}^{\prime} .
$$

Then the displayed equations asserted in (3) will follow immediately.

As in the proof of Proposition 3.1 $\left(2^{\prime}\right)$, the Laplace identity (2.4) yields

$$
F_{2}(\Delta) \subset F_{1}^{2}
$$

Furthermore, as in the proof of Lemma 2.5(3), the Laplace identity yields

$$
m_{1, k}^{j, 1} \Delta=m_{j}^{1} m_{k}^{1}-\alpha m_{k}^{j}
$$

because $\varphi$ is symmetric. Hence,

$$
I^{\prime 2} \subset F_{2}^{\prime}(\Delta)+(\alpha) F_{1}
$$

Since $F_{1}=I^{\prime}$ by $(1)$, therefore

$$
F_{2} \subset F_{1}^{2}:(\Delta)=I^{\prime 2}:(\Delta) \subset\left(F_{2}^{\prime} \Delta, \alpha\right):(\Delta) .
$$

Now, it follows easily from the definitions that

$$
\left(F_{2}^{\prime} \Delta, \alpha\right):(\Delta) \subset((\alpha):(\Delta))+F_{2}^{\prime} .
$$

However, since $\alpha, \Delta$ form a regular sequence,

$$
((\alpha):(\Delta))=(\alpha) .
$$

Together, the last three displays yield that

$$
F_{2} \subset F_{1}^{2}:(\Delta) \subset(\alpha)+F_{2}^{\prime}=F_{2}^{\prime} .
$$

Thus (3.6.1) holds, and so (3) is proved. 
Theorem 3.7. Let $R$ be a Noetherian local ring. Given an element $\Delta \in R$, set $A:=R /(\Delta)$ and let ${ }^{-}{ }^{\text {, }}$ indicate the image in $A$, and let $K$ denote the total ring of quotients of $A$. Given a finitely generated $R$-module $B$, set $F_{i}:=\operatorname{Fitt}_{i}^{R}(B)$. Then there is a bijective correspondence between the following two sets:

$\left\{B \mid B\right.$ a perfect $R$-algebra of grade 1 with grade $F_{1}=2$ and grade $\left.F_{2} \geq 3\right\}$, $\{(\Delta) \subset I \mid I$ a perfect $R$-ideal of grade 2 that is a complete intersection at each associated prime, and $\Delta \in I^{(2)}$ an $R$-regular element $\}$.

The correspondence associates to $B$ the pair $(\Delta):=F_{0}$ and $I:=F_{1}$, and conversely to $(\Delta) \subset I$ the $R$-module $B:=A:_{K} \bar{I}$; moreover, $A \subset B \subset K$ and $\bar{I}=A:_{K} B$. Finally, $B$ is Gorenstein if and only if $I=(\Delta, \alpha): I$ for some $\alpha \in I$; if so, then $B=\bar{\alpha}^{-1} \bar{I}$.

Proof. The assertions follow immediately from Propositions 3.1 and 3.6(3).

Proposition 3.8. Let $R$ be a Noetherian local ring, and $B$ a Gorenstein $R$-algebra. Assume that, as an $R$-module, $B$ is finitely generated and perfect of grade 1 . Set $F_{i}:=\operatorname{Fitt}_{i}^{R}(B)$. Then either height $F_{2} \leq 3$ or $F_{2}=R$.

In addition, assume grade $F_{1} \geq 2$ and grade $F_{2} \geq 3$. Then either $F_{2}$ is a perfect $R$-ideal of grade 3 , or it's the unit ideal.

Finally, let $\Delta$ be a generator of $F_{0}$, and set $I:=F_{1}$. Then $\Delta \in I^{(2)}$, and $\Delta$ defines an isomorphism,

$$
R / F_{2} \stackrel{\sim}{\longrightarrow} I^{(2)} / I^{2} .
$$

Proof. Proposition 2.9(1) yields height $F_{2} \leq 3$. Proposition 3.6(3) yields a symmetric matrix $\phi^{\prime}$ whose ideal of submaximal minors is equal to $F_{2}$. Hence $F_{2}$ is a perfect $R$-ideal of grade 3 since grade $F_{2} \geq 3$; see [24, Thm. 1, p. 116] for instance. Finally, by Proposition 3.6(2) and (3), the relation $\Delta \in I^{(2)}$ holds and the hypotheses of Proposition 3.3 obtain. So (3.3.3) yields $I^{(2)}=I^{2}+(\Delta)$. Hence, multiplication by $\Delta$ induces a surjection $R \rightarrow I^{(2)} / I^{2}$. Obviously, its kernel is $I^{2}:(\Delta)$. However, the latter is equal to $F_{2}$ by Proposition 3.6(3).

Corollary 3.9. Let $f: X \rightarrow Y$ be a finite map of locally Noetherian schemes. Assume that $f$ is locally of flat dimension 1 and Gorenstein. Then each component of $N_{3}$, the scheme of target triple points, has codimension at most 3.

Assume in addition that each component of $N_{3}$ has codimension 3 , that $f$ is birational onto its image, and that $Y$ satisfies Serre's condition $\left(\mathrm{S}_{3}\right)$. Let $\mathcal{I}_{N_{2}}$ denote the $\mathcal{O}_{Y}$-ideal of the scheme of target double-points, and $\mathcal{I}_{N_{2}}^{(2)}$ its second symbolic power. Then $\mathcal{O}_{N_{3}}$ is a perfect $\mathcal{O}_{Y}$-module, and $\mathcal{I}_{N_{2}}^{(2)} / \mathcal{I}_{N_{2}}^{2}$ is an invertible $\mathcal{O}_{N_{3}}$ module.

Proof. The assertions are local on $Y$. Furthermore, $\mathcal{O}_{Y}$ is perfect of grade 1 by virtue of [22, Lemma 2.3], and if $f$ is birational onto its image and $Y$ satisfies $\left(\mathrm{S}_{2}\right)$, then $\mathcal{I}_{N_{2}}$ has grade at least 2 by Lemma 2.11. So the assertions follow from Proposition 3.8 .

\section{Normality}

In this section, we study the normality of the algebra $B$ treated in Proposition 3.1 and Theorem 3.7. In other words, we investigate when $B$ is equal to the integral closure $A^{\prime}$ of $A$ in its total ring of quotients $K$. 
Given a Noetherian local ring $R$, let $e(R)$ denote the multiplicity of $R$, and call $R$ a hypersurface ring if $R \cong S /(x)$, where $S$ is a regular local ring and $x$ is an $S$-regular element. Given an ideal $I$ and an element $x$, set

$$
O(I):=\sup \left\{i \mid I \subset m^{i}\right\} \text { and } o(x):=O((x)),
$$

where $m$ is the maximal ideal, and call these numbers the orders of $I$ and $x$.

Proposition 4.1. Let $R$ be a Noetherian local ring satisfying $\left(\mathrm{R}_{2}\right)$ and $\left(\mathrm{S}_{3}\right)$. Let $B$ be an $R$-algebra that, as an $R$-module, is finitely generated and perfect of grade 1 , and assume that one of the equivalent conditions of Lemma 2.11 is satisfied. Set $F_{i}:=\operatorname{Fitt}_{i}^{R}(B)$ and $I:=F_{1}$. Set $A:=R / F_{0}$ and let $\Delta$ be a generator of $F_{0}$. Consider the following conditions:

(i) $e\left(A_{p}\right)=2$ for every associated prime $p$ of $I$, and $\Delta \in I^{(2)}$;

(ii) $e\left(A_{p}\right) \leq 3$ for every associated prime $p$ of $I$, and $\Delta \in I^{(2)}$;

(iii) $(R / I)_{p}$ is a hypersurface ring for every associated prime $p$ of $I$;

(iv) $I_{p}$ is a complete intersection for every associated prime $p$ of $I$;

(v) $\Delta \in I^{(2)}$.

Then (i) $\Rightarrow($ ii $) \Rightarrow($ iii $) \Rightarrow(\mathrm{iv}) \Rightarrow(\mathrm{v})$, and all five conditions are equivalent if $B$ is normal.

Proof. Localizing at an associated prime of $I$, we may assume that $R$ is a regular local ring of dimension 2 , that $I$ is primary for the maximal ideal $m$, and that $I^{(2)}=I^{2}$. First notice that $e\left(A_{p}\right) \geq 2$, because otherwise $A$ would be regular, and hence, since the extension $A \subset B$ is finite and birational, $A$ would be equal to $B$, contrary to the assumption that $I$, or $F_{1}$, is $m$-primary, so unequal to $R$.

Trivially, (i) implies (ii). Assume (ii). If $I \subset m^{2}$, then $\Delta \in I^{2} \subset m^{4}$ and so $e(A)=o(\Delta) \geq 4$. It follows that (iii) holds. Clearly, (iii) implies (iv). By Lemma 3.6(1), the hypotheses of Proposition 3.1 obtain, and its Part (3) yields (iv) $\Rightarrow(\mathrm{v})$.

Finally, suppose that $B$ is normal and that $\Delta$ is in $I^{(2)}$, so in $I^{2}$. We have to prove that $e(A)=2$. Set $\bar{I}:=I /(\Delta)$. Then $\bar{I}$ is equal to the conductor in $A$ of $B$ by Proposition 3.1(1). So, since $B$ is one-dimensional and normal, $\bar{I} \cong B$. Since $\Delta \in m I$, therefore

$$
I / m I \cong \bar{I} / m \bar{I} \cong B / m B
$$

Now, the length of $I / m I$ is equal to the minimal number of generators $\nu(I)$ of $I$, and the length of $B / m B$, viewed as an $A$-module, is equal to the multiplicity of $e(A)$. Hence

$$
\nu(I)=e(A)=o(\Delta) .
$$

On the other hand, the Hilbert-Burch theorem immediately yields the (well-known) inequality, $O(I) \geq \nu(I)-1$. Also, $o(\Delta) \geq 2 O(I)$ since $\Delta \in I^{2}$. So

$$
o(\Delta) \geq 2 O(I) \geq 2 \nu(I)-2 \geq \nu(I) .
$$

Together, the two preceding displays give $\nu(I)=2 \nu(I)-2$. So $\nu(I)=2$, and the proof is complete.

Example 4.2. Proposition 4.1 shows that, in general, the five conditions of Proposition 3.1(2) are not equivalent. Indeed, let $R$ be a power series ring in two variables over a field (of any characteristic), and let $\Delta$ be any reduced power series of order $o(\Delta)$ at least 3 , and set $A:=R /(\Delta)$. Let $B$ be the integral closure of $A$ in its total 
ring of quotients. Then, by Lemma 2.11 and Proposition 3.6, the hypotheses of Proposition 3.1 obtain. However, $\Delta \notin I^{(2)}$ by virtue of Proposition 4.1, although $B$ is a ring. For instance, say $R:=k[[x, y]]$ and $\Delta:=y^{3}-x^{4}$; then $I=\left(x^{2}, x y, y^{2}\right)$ because, if $B=k[[t]]$ say, then $A=k\left[\left[t^{3}, t^{4}\right]\right]$ and $I /(\Delta)=\left(t^{6}, t^{7}, t^{8}\right)$.

Lemma 4.3. Let $k$ be an infinite field, $L$ a field containing $k$. Let $\psi$ be an $s$ by $r$ matrix with coefficients in $L$ and with rank $s$. Then a general $k$-linear combination of the columns of $\psi$ does not satisfy any given nonzero polynomial $F$ in $L\left[X_{1}, \ldots, X_{s}\right]$.

Proof. Consider the polynomial $G:=F \circ \psi$ in $L\left[Y_{1}, \ldots, Y_{r}\right]$. Obviously, $G \neq 0$ because rank $\psi=s$ and $F \neq 0$. Let $\left\{a_{i}\right\}$ be a basis for $L / k$, and say $G=\sum a_{i} G_{i}$ where $G_{i} \in k\left[Y_{1}, \ldots, Y_{r}\right]$. Then $G_{i} \neq 0$ for some $i$, because $G \neq 0$. Hence, since $k$ is infinite, there exists a $y \in k^{r}$ such that $G_{i}(y) \neq 0$ for some $i$. On the other hand, for any such $y$, clearly $G(y) \neq 0$. Thus the assertion holds.

Theorem 4.4. Let $k$ be an infinite perfect field, and $R$ a Noetherian local $k$-algebra satisfying $\left(\mathrm{R}_{2}\right)$ and $\left(\mathrm{S}_{3}\right)$. Let $I$ be a perfect ideal of grade 2 that is a complete intersection at each associated prime. Fix generators $h_{1}, \ldots, h_{l}$ of $I^{(2)}$, and generators $x_{1}, \ldots, x_{n}$ of the maximal ideal of $R$. Let $f_{1}, \ldots, f_{r}$ be the sequence $h_{1}, \ldots, h_{l}$ if char $k=0$, and the sequence $x_{0} h_{1}, \ldots, x_{n} h_{l}$ with $x_{0}:=1$ if char $k$ is arbitrary. Let $\Delta$ be a general $k$-linear combination of $f_{1}, \ldots, f_{r}$, and set $A:=R /(\Delta)$. Let $K$ be the total ring of quotients of $A$, and set $B:=A:_{K} \bar{I}$. Then $B$ is a ring, and the following conditions are equivalent:

(i) $e\left(A_{p}\right)=2$ for every associated prime $p$ of $I$;

(ii) $e\left(A_{p}\right) \leq 3$ for every associated prime $p$ of $I$;

(iii) $(R / I)_{p}$ is a hypersurface ring for every associated prime $p$ of $I$;

(iv) $B$ is normal.

Furthermore, if any of these conditions hold, then the extension $B / A$ is unramified in codimension 1.

Proof. First of all, $B$ is a ring by Proposition 3.1(2), and $B$ is a perfect $R$-module of grade 1 by Proposition 3.1(1). So we may apply Proposition 4.1. Hence, (i) $\Rightarrow\left(\right.$ ii) $\Rightarrow$ (iii); moreover, (iv) $\Rightarrow\left(\right.$ i) since $\Delta \in I^{(2)}$. Finally, assume (iii). We have to prove that $B$ is normal and $B / A$ is unramified in codimension 1 .

By hypothesis, $R$ satisfies $\left(\mathrm{S}_{3}\right)$. Since $B$ is a perfect $R$-module of grade 1 , it is locally of codimension 1 by $[22,(2.3)$ and $(2.5)]$. Hence $B$ satisfies $\left(\mathrm{S}_{2}\right)$ as an $R$-module, and so as a ring. Therefore, it suffices to prove this: for every prime $p$ of $R$ with $p \ni \Delta$ and with $\operatorname{dim} R_{p} \leq 2$, the localization $B_{p}$ is regular and is unramified over $A_{p}$.

If $p \not \supset I$, then $A_{p}$ is regular by a form of Bertini's theorem since $R_{p}$ is regular and since $\Delta \in I^{(2)}$ is general. Indeed, in characteristic zero, the required form is [11, Thm. 4.6, p. 107]; in arbitrary characteristic, the required form follows from the proof of $[11$, Thm. 4.1, p. 106] and [11, Thm. 1.7, p. 101]. Thus we may assume that $p \supset I$. Then $p$ is one of the finitely many associated primes of $I$.

Localize at $p$. Then $R$ is a regular local ring of dimension 2 , and $I \not \subset m^{2}$, where $m$ is the maximal ideal, because $R / I$ is a hypersurface ring. Completing $R$, we may even suppose that $R=L[[x, y]]$, where $L$ is an extension field of $k$ and $x, y$ are indeterminates with $x \in I$. Then $I=\left(x, y^{s}\right)$ for some $s \geq 1$. Set $\bar{I}:=I /(\Delta)$. Let $\Delta_{x}$ stand for the partial derivative of $\Delta$ with respect to $x$, and $D$ for the image of 
$\Delta_{x}$ in $A$. We are now going to establish the following equation:

$$
D \bar{I}=\bar{I}^{2} \text {. }
$$

Since both $f_{1}, \ldots, f_{r}$ and $x^{2}, x y^{s}, y^{2 s}$ generate $I^{2}$, there exist a 3 by $n$ matrix $\varphi$ and an $n$ by 3 matrix $\psi$, both with entries in $R$, so that

$$
\left(\begin{array}{c}
x^{2} \\
x y^{s} \\
y^{2 s}
\end{array}\right)=\varphi\left(\begin{array}{c}
f_{1} \\
\vdots \\
f_{r}
\end{array}\right) \text { and }\left(\begin{array}{c}
f_{1} \\
\vdots \\
f_{r}
\end{array}\right)=\psi\left(\begin{array}{c}
x^{2} \\
x y^{s} \\
y^{2 s}
\end{array}\right) .
$$

Since $x^{2}, x y^{s}, y^{2 s}$ form a minimal generating set, $\varphi \psi$ is congruent to the 3 by 3 identity matrix modulo $m$. Hence $\psi$ has rank 3 modulo $m$; in other words,

$$
\operatorname{rank}_{L} \psi(0)=3 \text {, }
$$

where $\psi(0)$ is the matrix of constant terms. By definition,

$$
\Delta:=a_{1} f_{1}+\cdots+a_{r} f_{r},
$$

where $\left(a_{1}, \ldots, a_{r}\right)$ is a general point in $\mathbf{A}_{k}^{n}(k)$. Set

$$
(u, v, w):=\left(a_{1}, \ldots, a_{r}\right) \psi .
$$

Then the vector of constant terms $(u(0), v(0), w(0))$ is a general $k$-linear combination of the rows of $\psi(0)$; hence, it does not satisfy any given nonzero polynomial with coefficients in $L$, by Lemma 4.3 applied to the transpose of $\psi(0)$. On the other hand,

$$
\Delta=u x^{2}+v x y^{s}+w y^{2 s} .
$$

Set $\widetilde{u}:=2 u+x u_{x}$ and $\widetilde{v}:=v+x v_{x}+y^{s} w_{x}$. Then

$$
\Delta_{x}=\widetilde{u} x+\widetilde{v} y^{s} .
$$

Since $(u(0), v(0), w(0))$ satisfies no given nonzero polynomial, $w(0) \neq 0$ and

$$
\left(\widetilde{u}^{2} w-\widetilde{u} v \widetilde{v}+u \widetilde{v}^{2}\right)(0)=4 u^{2}(0) w(0)-u(0) v^{2}(0) \neq 0 .
$$

So $w$ and $\widetilde{u}^{2} w-\widetilde{u} v \widetilde{v}+u \widetilde{v}^{2}$ are units in $R$. Since $w$ is a unit,

$$
\begin{aligned}
\left(\Delta, \Delta_{x} I\right) & =\left(u x^{2}+v x y^{s}+w y^{2 s},\left(\widetilde{u} x+\widetilde{v} y^{s}\right) x, w\left(\widetilde{u} x+\widetilde{v} y^{s}\right) y^{s}\right) \\
& =\left(u x^{2}+v x y^{s}+w y^{2 s}, \widetilde{u} x^{2}+\widetilde{v} x y^{s}, w \widetilde{u} x y^{s}-\widetilde{v}\left(u x^{2}+v x y^{s}\right)\right) \\
& =\left(u x^{2}+v x y^{s}+w y^{2 s}, \widetilde{u} x^{2}+\widetilde{v} x y^{s},-\widetilde{v} u x^{2}+(w \widetilde{u}-\widetilde{v} v) x y^{s}\right) .
\end{aligned}
$$

However,

$$
\operatorname{det}\left(\begin{array}{cc}
\widetilde{u} & \widetilde{v} \\
-\widetilde{v} u & (w \widetilde{u}-\widetilde{v} v)
\end{array}\right)=\widetilde{u}^{2} w-\widetilde{u} v \widetilde{v}+u \widetilde{v}^{2},
$$

and the latter is a unit. Hence

$$
\left(\Delta, \Delta_{x} I\right)=\left(\Delta, x^{2}, x y^{s}\right)=\left(\Delta, x^{2}, x y^{s}, y^{2 s}\right)=\left(\Delta, I^{2}\right) .
$$

So (4.4.1) holds.

Since $D \bar{I}=\bar{I}^{2}$ by (4.4.1), Proposition 3.1(4) yields that $\bar{I}=D B$ and that $D$ is a nonzerodivisor. The latter implies that the extension $A / L[[y]]$ is generically unramified. Hence $A$ is generically reduced, so reduced since it has no embedded components. Therefore, the integral closure $A^{\prime}$ of $A$ in $K$ is a finitely generated $A$-module. 
Let $C$ denote the conductor. Then $C$ contains $D$, and $C=D A^{\prime}$ if and only if $A^{\prime} / L[[y]]$ is unramified; this is a matter of standard theory, see [23, Cor. G.12, p. 389] and [23, Cor. G.14c), p. 391] for example. On the other hand, $B \subseteq A^{\prime}$ since $B$ is a finitely generated $A$-module; hence, $C \subseteq \bar{I}$ because $\bar{I}=A:_{K} B$ by Proposition 3.1(1). Therefore,

$$
C \subseteq \bar{I}=D B \subseteq D A^{\prime} \subseteq C .
$$

So $D B=D A^{\prime}$, whence $B=A^{\prime}$, and thus $B$ is normal. Moreover, $D A^{\prime}=C$, whence $A^{\prime} / L[[y]]$ is unramified, and so $A^{\prime} / A$ is too. The proof is now complete.

Example 4.5. In Theorem 4.4, it is necessary to assume that $\Delta$ is general. For example, let $k$ be a field of any characteristic, and $R:=k[[x, y]]$ a power series ring. Set $I:=(x, y)$ and $\Delta:=x^{2}-y^{5}$. Then $A^{\prime}$ is a power series ring in one variable, say $A^{\prime}=k[[t]]$, and $A$ and $B$ are subrings; namely, $A=k\left[\left[t^{5}, t^{2}\right]\right]$ and $B=k\left[\left[t^{3}, t^{2}\right]\right]$. So $\bar{I}=\left(t^{5}, t^{2}\right)$ and $C=\left(t^{5}, t^{4}\right)$. Moreover, $D=t^{5}$ if $\operatorname{char} k \neq 2$, and $D=0$ if char $k=2$.

\section{ACKNOWLEDGEMENTS}

It is a pleasure to thank David Mond for many stimulating discussions. It is a pleasure to thank David Eisenbud for his comments, which led to the present improved versions of 2.1 and 2.2. It is a pleasure to thank Hubert Flenner for his help with Bertini's theorem for local rings. Finally, it is a pleasure to thank Joseph Lipman for his long collaboration on the present subject; in the estimation of the two authors de jure, if not in his own, he is a third author de facto.

\section{REFERENCES}

1. M. Artin and M. Nagata, Residual intersections in Cohen-Macaulay rings, J. Math. Kyoto Univ. 121972 307-23. MR 46:166

2. M. Brodmann, The asymptotic nature of analytic spread, Math. Proc. Camb. Phil. Soc. 86 1979 35-39. MR 81e:13033

3. W. Bruns, The Eisenbud-Evans generalized principal ideal theorem and determinantal ideals, Proc. Amer. Math. Soc. 831981 19-24. MR 82k:13010

4. D. Buchsbaum and D. Eisenbud, What annihilates a module? J. Algebra 471977 231-43. MR 57:16293

5. D. Buchsbaum and D. Eisenbud, Algebraic structures for finite free resolutions, and some structure theorems for ideals of codimension 3, Amer. J. Math. 991977 447-85. MR 56:11983

6. F. Catanese, Commutative algebra methods and equations of regular surfaces, Algebraic Geometry, Bucharest 1982, L. Bădescu and D. Popescu (eds.), Lecture Notes in Math. 1056, Springer-Verlag, 1984, pp. 68-111. MR 86c:14027

7. T. de Jong and D. van Straten, Deformations of the normalization of hypersurfaces, Math. Ann. 2881990 527-47. MR 92d:32050

8. J. Eagon and D. Northcott, Ideals defined by matrices and a certain complex associated to them, Proc. Royal Soc. A269 1962 188-204. MR 26:161

9. D. Eisenbud, Homological algebra on a complete intersection, with an application to group representations, Trans. Amer. Math. Soc. 2601980 35-64. MR 82d:13013

10. D. Eisenbud and B. Mazur, Symbolic squares, Fitting ideals, and evolutions, Preprint.

11. H. Flenner, Die Sätze von Bertini für lokale Ringe, Math. Ann. 229 1977 97-111. MR 57:311

12. S. Goto and K. Nishida, The Cohen-Macaulay and Gorenstein Rees algebras associated to filtrations, Mem. Amer. Math. Soc., vol. 110, 1994, no. 526. MR 95b:13001

13. S. Goto, K. Nishida, and Y. Shimoda, The Gorensteinness of symbolic Rees algebras for space curves, J. Math. Soc. Japan 431991 465-81. MR 92h:13019

14. S. Goto, K. Nishida, and K. Watanabe, Non-Cohen-Macaulay symbolic blow-ups for space monomial curves and counterexamples to Cowsik's question, Proc. Amer. Math. Soc. 120 1994 383-92. MR 94d:13005 
15. M. Grassi, Koszul modules and Gorenstein algebras, J. Algebra 1801996 918-53. MR 96b: 13021

16. A. Grothendieck, Cohomologie locale des faisceaux cohérents et théorèmes de Lefschetz locaux et globaux, (SGA II), Exposé XIV, Advanced Studies in Math, Vol. II, North Holland Publishing Co., Amsterdam, 1968. MR 57:16294

17. J. Herzog and M. Kühl, Maximal Cohen-Macaulay modules over Gorenstein rings and Bourbaki sequences, in "Commutative Algebra and Combinatorics," M. Nagata and H. Matsumura (eds.), Advanced Studies in Pure Math 11, North-Holland, Amsterdam (1987), 65-92. MR 89h:13029

18. J. Herzog and B. Ulrich, Self-linked curve singularities, Nagoya Math J. 1201990 129-53. MR 92c: 13010

19. T. Jozéfiak, Ideals generated by minors of a symmetric matrix, Comment. Math. Helv. 53 1978 594-607. MR 80g:14041

20. T. Jozéfiak and P. Pragacz, Ideals generated by Pfaffians, J. Algebra 611979 189-98. MR 81e:13005

21. S. Kleiman, J. Lipman, and B. Ulrich, The source double-point cycle of a finite map of codimension one, in "Complex Projective Varieties," G. Ellingsrud, C. Peskine, G. Sacchiero, and S. A. Stromme (eds.), London Math. Soc. Lecture Note Series 1791992 199-212. MR 94a:14003

22. S. Kleiman, J. Lipman, and B. Ulrich, The multiple-point schemes of a finite curvilinear map of codimension one, Ark. Mat. 341996 285-326. CMP 97:03

23. E. Kunz, Kähler differentials, Advandced Lectures in Math., Vieweg, 1986. MR 88e:14025

24. R. Kutz, Cohen-Macaulay rings and ideal theory in rings of invariants of algebraic groups, Trans. Amer. Math. Soc. 1941974 115-29. MR 50:4570

25. H. Matsumura, Commutative ring theory, Cambridge Studies in Advanced Math 8, 1986. MR 88h:13001

26. D. Mond and R. Pellikaan, Fitting ideals and multiple points of analytic mappings, Algebraic geometry and complex analysis, E. Ramírez de Arellano (ed.), Proc. Conf., Pátzcuaro 1987, Lecture Notes in Math. 1414, Springer-Verlag, 1989, pp. 107-161. MR 91e:32035

27. I. Reiten, The converse of a theorem of Sharp on Gorenstein modules, Proc. Amer. Math. Soc. 401972 417-20. MR 45:5128

28. P. Roberts, A prime ideal in a polynomial ring whose symbolic blow-up is not Noetherian, Proc. Amer. Math. Soc. 941985 589-92. MR 86k:13017

29. P. Roberts, An infinitely generated symbolic blow-up in a power series ring and a new counterexample to Hilbert's fourteenth problem, J. Algebra 1321990 461-73. MR 91j:13006

30. J. Shamash, The Poincaré series of a local ring, J. Algebra 121969 453-70. MR 39:2751

31. P. Valabrega and G. Valla, Form rings and regular sequences, Nagoya Math J. 72 1978 93-101. MR 80d:14010

32. G. Valla, On set-theoretic complete intersections, Complete Intersections, S. Greco and R. Strano (eds.), Proc. Conf., Arcireale 1983 Lecture Notes in Math. 1092, Springer-Verlag, 1984, pp. 85-101. MR 86f: 14033

Department of Mathematics, Room 2-278, Massachusetts Institute of Technology, Cambridge, Massachusetts 02139-4307

E-mail address: Kleiman@math.MIT.edu

Department of Mathematics, Michigan State University, East Lansing, Michigan 48824-1027

E-mail address: Ulrich@math.MSU.edu 